\title{
Mapping class group of a handlebody
}

by

\author{
Bronisław W a j n r y b (Haifa)
}

\begin{abstract}
Let $B$ be a 3-dimensional handlebody of genus $g$. Let $\mathcal{M}$ be the group of the isotopy classes of orientation preserving homeomorphisms of $B$. We construct a 2-dimensional simplicial complex $X$, connected and simply-connected, on which $\mathcal{M}$ acts by simplicial transformations and has only a finite number of orbits. From this action we derive an explicit finite presentation of $\mathcal{M}$.
\end{abstract}

We consider a 3-dimensional handlebody $B=B_{g}$ of genus $g>0$. We may think of $B$ as a solid 3-ball with $g$ solid handles attached to it (see Figure 1). Our goal is to determine an explicit presentation of the mapping class group of $B$, the group $\mathcal{M}_{g}$ of the isotopy classes of orientation preserving homeomorphisms of $B$. Every homeomorphism $h$ of $B$ induces a homeomorphism of the boundary $S=\partial B$ of $B$ and we get an embedding of $\mathcal{M}_{g}$ into the mapping class group $\operatorname{MCG}(S)$ of the surface $S$.

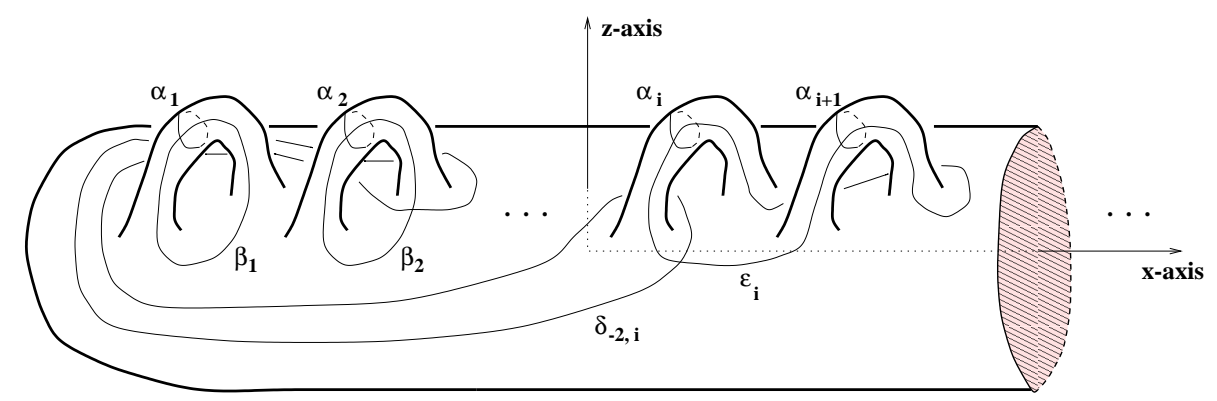

Fig. 1. Handlebody

An explicit and quite simple presentation of $\operatorname{MCG}(S)$ is now known, but it took a lot of time and effort of many people to reach it (see [1], [3], [12],

1991 Mathematics Subject Classification: 20F05, 20F38, 57M05, 57M60.

This research was partially supported by the fund for the promotion of research at the Technion. 
[9], [7], [11], [6], [14]). The main step towards an explicit presentation of $\operatorname{MCG}(S)$ was done by Hatcher and Thurston in [7] where they constructed a connected and simply-connected cellular complex-a cut-system complex of $S$ - on which $\operatorname{MCG}(S)$ acts by cellular transformations with a finite number of orbits and well understood stabilizers. A presentation of $\operatorname{MCG}(S)$ was obtained from this action by methods described more explicitly by Laudenbach [11]. The presentation was simplified by Harer [6], and further simplified by Wajnryb [14].

No presentation of $\mathcal{M}_{g}$ has been known until now. A finite set of generators for $\mathcal{M}_{g}$ was first obtained by Suzuki [13]. It was quite similar to the set of generators in our Theorem 18, the main result of this paper. The group $\mathcal{M}_{g}$ has an infinite index in $\operatorname{MCG}(S)$ so the knowledge of $\operatorname{MCG}(S)$ does not help directly in the investigation of $\mathcal{M}_{g}$. However, we apply the main idea of Hatcher and Thurston and construct a simplicial, 2-dimensional cut-system complex $X$ of $B$, similar to the cut-system complex of $S$, on which $\mathcal{M}_{g}$ acts by simplicial transformations. We prove by a direct method, quite different from the method of Hatcher and Thurston, that $X$ is connected and simply-connected. We describe the orbits of the action of $\mathcal{M}_{g}$ on vertices, edges and faces of $X . \mathcal{M}_{g}$ acts transitively on vertices and has only a finite number of edge-orbits and face-orbits. We describe the stabilizer of a vertex and the stabilizer of each edge and then apply the ideas from [7] and [11] to obtain an explicit presentation of $\mathcal{M}_{g}$ (Theorem 18). We follow the Master dissertation of Michael Heusner [8] in which the method of [7] and [11] was very clearly and precisely explained. Unfortunately, an explicit presentation of $\mathcal{M}_{g}$ (Theorem 18) obtained in this way is still rather long and complicated.

1. Cut-system complex. In this section we construct the complex $X$ and prove that it is connected and simply-connected.

Let $B$ be a handlebody of genus $g$ and let $S$ be the boundary of $B$. By a curve on $S$ we always mean a simple closed curve. A curve $\alpha$ is a meridian curve if it bounds a disk $D$ in $B$ such that $D \cap S=\partial D=\alpha$. Then $D$ is called a meridian disk. If $\alpha$ is a meridian curve, non-separating on $S$, and if we cut $B$ along a corresponding meridian disk $D$, we get a handlebody $B^{\prime}$ of genus $g-1$. On the boundary of $B^{\prime}$ we have two copies of $D$ - two distinguished disks. For the purpose of induction on genus we shall consider a handlebody $B$ with a finite number of disjoint distinguished disks on its boundary $S$ and we shall assume that all homeomorphisms and all isotopies of $S$ are fixed on the distinguished disks. If we have disjoint meridian curves on $S$ we can construct pairwise disjoint meridian disks.

We start with disks $D_{1}$ and $D_{2}$ and put $D_{2}$ into general position with respect to $D_{1}$. Then they intersect along disjoint circles. Consider an inner- 
most circle $C$ on $D_{1}$. It bounds a disk $A_{1}$ in $D_{1}$. It also bounds a disk $A_{2}$ in $D_{2}$. Since $C$ is innermost on $D_{1}$ the disk $A_{1}$ does not meet $D_{2}$ outside $C$. In particular $A_{1}$ and $A_{2}$ meet only at their common boundary $C$. Their union forms a sphere. Since $B$ is irreducible the sphere bounds a 3-ball. We can push $A_{2}$ across the 3 -ball off $A_{1}$. By induction we can get rid of all the intersection of $D_{1}$ and $D_{2}$. Now we keep $D_{1}$ and $D_{2}$ fixed and deform $D_{3}$ to make it disjoint from the other two disks, and so on.

A cut-system on $S$ is an isotopy class of an unordered collection of $g$ disjoint meridian curves $\alpha_{1}, \ldots, \alpha_{g}$ which are linearly independent in the homology group $H_{1}(B)$ and are disjoint from the distinguished disks. When we cut $B$ open along the corresponding disjoint meridian disks we get a 3 ball. We denote a cut-system by $\left\langle\alpha_{1}, \ldots, \alpha_{g}\right\rangle$. We say that two cut-systems are related by a simple move if they have $g-1$ curves in common and the other two curves are disjoint. To simplify notation we drop the symbols for unchanging circles, e.g. $\left\langle\alpha_{i}\right\rangle \rightarrow\left\langle\alpha_{i}^{\prime}\right\rangle$ means that the curve $\alpha_{i}$ of a cut-system is replaced by a meridian curve $\alpha_{i}^{\prime}$ and the other $g-1$ curves of the cut-system do not change. It also means that $\alpha_{i}^{\prime}$ is disjoint from all the curves of the cut-system and that the new collection of curves forms a new cut-system.

We construct a 2-dimensional complex $X$. The vertices of $X$ are the cutsystems on $S$. Two cut-systems are connected by an edge if they are related by a simple move. If three vertices of $X$ have $g-1$ curves in common and the three remaining curves, one from each cut-system, are pairwise disjoint, then each pair of the vertices is connected by an edge in $X$ and the vertices form a triangle, a closed edge-path with three edges. We glue a face to every triangle and get a 2-dimensional simplicial complex $X$. The complex $X$ is called the cut-system complex of the handlebody $B$.

The main result of this section is the following

THEOREM 1. The cut-system complex $X$ is connected and simply-connected.

A homeomorphism $h$ of $B$ onto itself takes a meridian disk to a meridian disk. It takes a cut-system to a cut-system. If we let $h\left\langle\alpha_{1}, \ldots, \alpha_{g}\right\rangle=$ $\left\langle h\left(\alpha_{1}\right), \ldots, h\left(\alpha_{g}\right)\right\rangle$ we get an action of the mapping class group $\mathcal{M}_{g}$ on the vertices of $X$. The action also takes vertices connected by an edge to vertices connected by an edge, so it is a simplicial action.

Every curve on $S$ is ambient-isotopic to a PL-curve (piecewise-linear). We may assume that all curves in this paper are PL and that in the given, finite collection of curves different curves intersect only in a finite number of points, transversely, and no point belongs to three or more curves. We denote the number of intersection points of $\alpha$ and $\beta$ by $|\alpha \cap \beta|$. We want to prove that $X$ is simply-connected. We start with a fixed closed edge-path and want to prove that it is null-homotopic in $X$, i.e. it decomposes into a 
sum of triangles. We fix a particular collection of curves representing each vertex and assume that all curves of all these collections intersect transversely at a finite number of points and no point belongs to more than two curves. We may later introduce new curves subject to the same conditions.

We first consider the case of genus $g=1$.

If $B$ is a solid torus than all non-trivial (not contractible on $S$ ) meridian curves on the boundary $S$ of $B$ are isotopic. A cut-system is an isotopy class of a single meridian curve and thus $X$ consists of a single point. But if there are distinguished disks on $S$, which we need for the induction purpose, then different meridian curves may be non-isotopic in the complement of the distinguished disks and they may intersect essentially. In that case the complex $X$ may be quite complicated.

We say that two meridian curves $\alpha$ and $\beta$ on $S$ form a 2-gon if there is a disk (a 2-gon) in $S$ bounded by an arc of $\alpha$ and an $\operatorname{arc}$ of $\beta$. A 2-gon may contain distinguished disks inside. A collection of curves forms a 2-gon if a pair of curves of this collection form a 2-gon. In that case a minimal 2 -gon is one which does not contain another 2-gon inside. If $\alpha$ and $\beta$ form a minimal 2-gon then any other curve of the collection crosses the 2-gon along arcs which meet each of the curves $\alpha$ and $\beta$ once. If two curves on $S$ are homotopic and not disjoint then they form a 2-gon (see [5]).

Lemma 2. If $g=1$ then $X$ is connected.

P r o of. Let $\alpha$ and $\beta$ be two meridian curves on $S$. They are homotopic, so if they are not disjoint they form a 2-gon. Consider a minimal 2-gon, say bounded by $\operatorname{arcs} a$ and $b$ of $\alpha$ and $\beta$ respectively. If we replace the $\operatorname{arc} a$ of $\alpha$ by the $\operatorname{arc} b$ we get a new curve $\alpha^{\prime}$ homotopic to $\alpha$ on $S$ (see Figure 2). Then $\alpha^{\prime}$ is a meridian curve, $\left|\alpha \cap \alpha^{\prime}\right|=0$ and $\left|\beta \cap \alpha^{\prime}\right|<|\beta \cap \alpha|$. It follows by induction on $|\alpha \cap \beta|$ that $\alpha$ and $\beta$ can be connected by a sequence of curves such that the consecutive curves are disjoint, and thus connected by an edge in $X$.

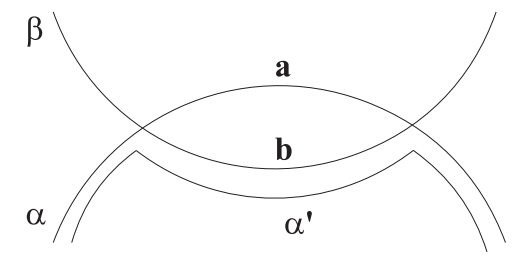

Fig. 2. The curves $\alpha$ and $\beta$ form a 2-gon

Lemma 3. Let $g=1$ and let $\mathbf{p}=\left\{\alpha_{1}, \ldots, \alpha_{k}\right\}$ be a closed edge-path in $X$, i.e. $\alpha_{1}=\alpha_{k}$. There exists another closed edge-path $\mathbf{p}^{\prime}=\left\{\alpha_{1}^{\prime}, \ldots, \alpha_{k}^{\prime}\right\}$ homotopic to $\mathbf{p}$ in $X$ and such that the collection of curves $\alpha_{1}^{\prime}, \ldots, \alpha_{k}^{\prime}$ forms no 2-gons. 
Pr o of. Consider a minimal 2-gon. Suppose it is formed by arcs $a$ and $b$ of curves $\alpha_{i}$ and $\alpha_{j}$ respectively. If we replace $b$ by $a$ (as in Figure 2) we get a new curve $\alpha_{j}^{\prime}$, homotopic to $\alpha_{j}$ on $S$ and disjoint from $\alpha_{j}$. So it is a meridian curve and represents a vertex in $X$. Also $\left|\alpha_{j}^{\prime} \cap \alpha_{i}\right|<\left|\alpha_{j} \cap \alpha_{i}\right|$. By a previous remark, $\alpha_{j}^{\prime}$ does not form any new intersections with other curves so it is also disjoint from $\alpha_{j-1}$ and $\alpha_{j+1}$ and is connected by edges to $\alpha_{j-1}, \alpha_{j}$, and $\alpha_{j+1}$. When we replace $\alpha_{j}$ by $\alpha_{j}^{\prime}$ we get a new edge-path and the difference between it and the old path is a sum of two triangles. Thus the new path is homotopic to $\mathbf{p}$ in $X$ and its curves intersect in a smaller number of points. Lemma 3 follows by induction.

Lemma 4. If $g=1$ then $X$ is simply-connected.

Proof. Consider a closed edge-path in $X$. We want to prove that it can be decomposed into a sum of triangles. By the previous lemma we may assume that it has no 2-gons. But meridian curves on a solid torus are homotopic. If there are no 2-gons then all curves are disjoint, and every pair is connected by an edge. In particular the edge-path is a sum of triangles as required.

From now on we assume that $B$ has genus $g$ greater than 1 .

InduCtion Hypothesis 1. The cut-system complex of a handlebody of genus less than $g$ is connected and simply-connected.

Our next task is to prove that $X$ is connected. We start with a lemma which will also be used later, in the proof that $X$ is simply-connected, so it may seem unnecessarily complicated at this stage.

Lemma 5. Let $\alpha, \beta, \gamma_{1}, \gamma_{2}$ be non-separating meridian curves on $S$ such that $\left|\gamma_{1} \cap \gamma_{2}\right|=k>0,\left|\gamma_{1} \cap \alpha\right|<n$, and $\left|\gamma_{2} \cap \alpha\right| \leq n$. Then there exists a non-separating meridian curve $\delta$ such that $\left|\delta \cap \gamma_{1}\right|<k,\left|\delta \cap \gamma_{2}\right|<k$, and $|\delta \cap \alpha|<n$. If also $\left|\gamma_{1} \cap \beta\right|=\left|\gamma_{2} \cap \beta\right|=0$, then $|\delta \cap \beta|=0$.

Proof. We may put the meridian disks $D_{1}$ and $D_{2}$, bounded by $\gamma_{1}$ and $\gamma_{2}$ respectively, into general position. Then they intersect along disjoint arcs and circles. We can get rid of the circles of intersection by moving disks across 3-balls corresponding to innermost circles. Consider now an innermost arc $d$ of the intersection (closest to the boundary) on $D_{1}$. The arc splits $D_{1}$ into two disks. One of them, say $D_{1}^{\prime}$, does not meet $D_{2}$ (see Figure 3). The disk $D_{1}^{\prime}$ is bounded by $d$ and by an $\operatorname{arc} c_{1}$ of $\gamma_{1}$. The $\operatorname{arc} c_{1}$ contains $m$ points of the curve $\alpha$. Consider also a corresponding construction for an innermost arc on $D_{2}$. Among all innermost arcs choose a "minimal" $\operatorname{arc} d$, one for which the number $m$ is minimal. If there are several such "minimal" arcs and one of them is innermost on $D_{2}$ we choose that one. 


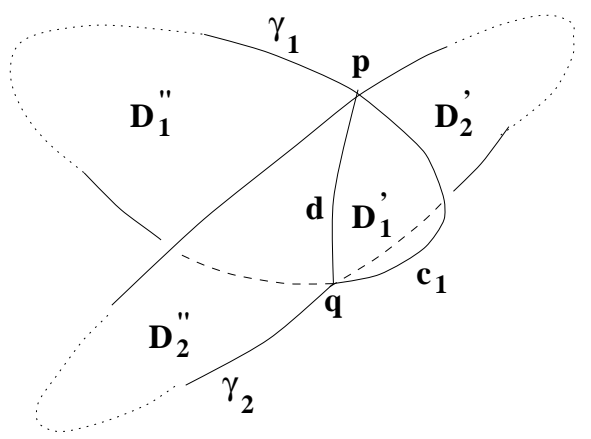

Fig. 3. Meridian disks of $\gamma_{1}$ and $\gamma_{2}$

Suppose first that the minimal arc $d$ is innermost on $D_{1}$ (see Figure 3 ). Let $p$ and $q$ be the end points of $d$. The arc $d$ also splits $D_{2}$ into two disks $D_{2}^{\prime}$ and $D_{2}^{\prime \prime}$ bounded by $d$ and by $\operatorname{arcs} c_{2}^{\prime}$ and $c_{2}^{\prime \prime}$ of $\gamma_{2}$ respectively. These disks may intersect $D_{1}$ but not inside $D_{1}^{\prime}$. When we replace the $\operatorname{arc} c_{2}^{\prime}$ (or $c_{2}^{\prime \prime}$ ) of $\gamma_{2}$ by $c_{1}$ we get a meridian curve $\delta^{\prime}$ (respectively $\delta^{\prime \prime}$ ) on $S$ which bounds a meridian disk $D_{1}^{\prime} \cup D_{2}^{\prime \prime}$ (respectively $D_{1}^{\prime} \cup D_{2}^{\prime}$ ). We can push $\delta^{\prime}\left(\right.$ or $\delta^{\prime \prime}$ ) off itself on $S$, away from the intersections $p$ and $q$, making it disjoint from $\gamma_{2}$. We get rid of the intersection points $p$ and $q$ at least, so $\left|\delta^{\prime} \cap \gamma_{1}\right|<k$ and $\left|\delta^{\prime \prime} \cap \gamma_{1}\right|<k$. In the first homology group of $S$ we have $\delta^{\prime \prime}+\delta^{\prime}=\gamma_{2}$. Since $\gamma_{2}$ is non-separating, i.e. non-trivial in the homology group, one of the curves must be non-separating and we choose this curve for $\delta$. The disks $D_{2}^{\prime}$ and $D_{2}^{\prime \prime}$ must contain innermost arcs (possibly $d$ is innermost on one or both disks), therefore both $c_{2}^{\prime}$ and $c_{2}^{\prime \prime}$ meet $\alpha$ more than $m$ times, by the minimality condition on $d$. We traded such an arc of $\gamma_{2}$ for the $\operatorname{arc} c_{1}$, therefore $|\delta \cap \alpha|<n$.

Suppose now that the minimal arc $d$ is innermost on $D_{2}$. We switch the roles of $D_{1}$ and $D_{2}$ (also in Figure 3 ) and repeat the construction of $\delta$. There may be some innermost arcs on $D_{1}$ for which the corresponding arc of $\gamma_{1}$ also meets $\alpha$ only $m$ times, but not less. In order to construct $\delta$ we replace an arc of $\gamma_{1}$ which meets $\alpha$ at least $m$ times by an arc of $\gamma_{2}$ which meets $\alpha$ exactly $m$ times. So $\delta$ meets $\alpha$ at most as many times as $\gamma_{1}$ and again $|\delta \cap \alpha|<n$ and the other properties of $\delta$ are as in the first case. The assertion about the curve $\beta$ follows from the construction.

Lemma 6. If $v_{1}$ and $v_{2}$ are vertices in $X$ which have one or more curves in common then they can be connected by an edge-path of vertices in $X$ all of which contain the common curves of $v_{1}$ and $v_{2}$.

Pr o of. When we cut $B$ open along the disjoint meridian disks bounded by the common curves of $v_{1}$ and $v_{2}$ we get a handlebody $B^{\prime}$ of a smaller genus and the remaining curves of $v_{1}$ and $v_{2}$ form two vertices of the cut-system 
complex of $B^{\prime}$. By Induction Hypothesis 1 there exists an edge-path $\mathbf{p}^{\prime}$ in this complex which connects the two vertices. When we add the common curves of $v_{1}$ and $v_{2}$ to curves of each vertex of the edge-path $\mathbf{p}^{\prime}$ we get cut-systems on $B$ which form an edge-path in $X$ connecting $v_{1}$ and $v_{2}$.

LEMMA 7. If $\alpha$ and $\beta$ are non-separating meridian curves then there exist vertices $v_{1}$ and $v_{2}$ of $X$ which are connected by an edge-path in $X$ (possibly $v_{1}=v_{2}$ ) and contain the curves $\alpha$ and $\beta$ respectively.

Proof. We prove the lemma by induction on $n=|\alpha \cap \beta|$. Assume first that the curves are disjoint. If $\alpha \cup \beta$ does not separate the surface $S$ then the pair $\alpha, \beta$ can be completed to a cut-system on $B$ representing a vertex $v_{1}=v_{2}$. Suppose now that $\alpha \cup \beta$ separates $S$. When we cut $B$ along disjoint meridian disks bounded by $\alpha$ and $\beta$ respectively we get two handlebodies $B_{1}$ and $B_{2}$. We choose a cut-system on each of the handlebodies. We get together $g-1$ meridian curves. When we add $\alpha$ or $\beta$ to these $g-1$ curves we get two cut-systems on $B$ which are related by a simple move.

Assume now that $n>0$. By Lemma 5 we can find a non-separating meridian curve $\gamma$ with $|\gamma \cap \alpha|<n$ and $|\gamma \cap \beta|<n$. By the induction hypothesis there exists a vertex $v_{1}$ of $X$ containing $\alpha$ and connected by an edge-path to a vertex $v_{3}$ containing $\gamma$. There also exists a vertex $v_{4}$ containing $\gamma$ and connected by an edge-path to a vertex $v_{2}$ containing $\beta$. Finally, $v_{3}$ and $v_{4}$ are connected by an edge-path by Lemma 6 .

As an easy corollary we get

Proposition 8. $X$ is connected.

Pr o of. If $v_{1}$ is a vertex of $X$ containing some curve $\alpha$ and $v_{2}$ is a vertex of $X$ containing some curve $\beta$ then, by Lemma 7 , there exist other vertices $v_{3}$ and $v_{4}$ connected by an edge-path and containing $\alpha$ and $\beta$ respectively. By Lemma 6 we can connect $v_{1}$ to $v_{3}$ and $v_{4}$ to $v_{2}$ by edge-paths in $X$.

We now proceed to prove that $X$ is simply-connected.

We define the distance between two curves $\alpha$ and $\beta$ as their intersection number $|\alpha \cap \beta|$. The distance between a curve $\gamma$ and a vertex $\left\langle\alpha_{1}, \ldots, \alpha_{g}\right\rangle$ is equal to the minimum of the distances $\left|\gamma \cap \alpha_{i}\right|$. The radius of an edge-path around a curve $\alpha$ is equal to the maximum of the distances from $\alpha$ to the vertices of the edge-path.

A segment is a sequence of consecutive vertices of an edge-path which have a curve in common. We say it is an $(\alpha, \beta, \ldots, \gamma)$-segment if all vertices of the segment contain the curves $(\alpha, \beta, \ldots, \gamma)$.

The general idea of the proof is to start with a closed edge-path in $X$ and reduce it to a sum of simpler edge-paths, and eventually to a sum of triangles, by induction on the radius of the edge-path around a fixed curve $\alpha$ and induction on the number of segments of maximal distance from $\alpha$. 
Lemma 9. A closed segment is null-homotopic.

P r o of. We cut $B$ open along the meridian disk bounded by the common curve $\alpha$ of the segment, getting a handlebody $B^{\prime}$ of a smaller genus. The remaining curves of each vertex form a vertex of a closed path in the cutsystem complex of $B^{\prime}$. This path decomposes into a sum of triangles by Induction Hypothesis 1 . When we add back the curve $\alpha$ to every vertex we get a decomposition of the initial closed segment into a sum of triangles.

LEMma 10. A closed path $\mathbf{p}=\langle\alpha, \gamma\rangle \rightarrow\langle\beta, \gamma\rangle \rightarrow\langle\beta, \delta\rangle \rightarrow\langle\alpha, \delta\rangle \rightarrow$ $\langle\alpha, \gamma\rangle$ is null-homotopic in $X$.

Pr o of. If $g>2$ then the path is a closed segment, all vertices of $\mathbf{p}$ have at least one additional common curve, so we may assume $g=2$. All the curves are disjoint. If $\alpha \cup \beta$ (or $\gamma \cup \delta$ ) does not separate $S$ there is a vertex $\langle\alpha, \beta\rangle$ (respectively $\langle\gamma, \delta\rangle$ ) which is connected by an edge to every vertex of the path and splits the path into a sum of four triangles. In the other case let us split $B$ open along the meridian disks of $\alpha, \beta, \gamma$, and $\delta$. Since $\alpha \cup \gamma$ does not separate $S$ we get three connected components and eight boundary components.

There is a connected component with at least three boundary components. By the separation properties of $\alpha, \beta, \gamma$ and $\delta$ this connected component has three boundary components corresponding to three different curves out of $\alpha, \beta, \gamma$ and $\delta$. Two of them belong to one vertex, say $\alpha$ and $\gamma$. We can form a connected sum $\alpha+\gamma$ of the two curves inside the connected component. We get a new meridian curve $\varepsilon$ disjoint from all four curves. The curve $\varepsilon$ forms a vertex with each of the four curves. The path $\mathbf{p}$ decomposes into a sum of ten triangles (see Figure 4).

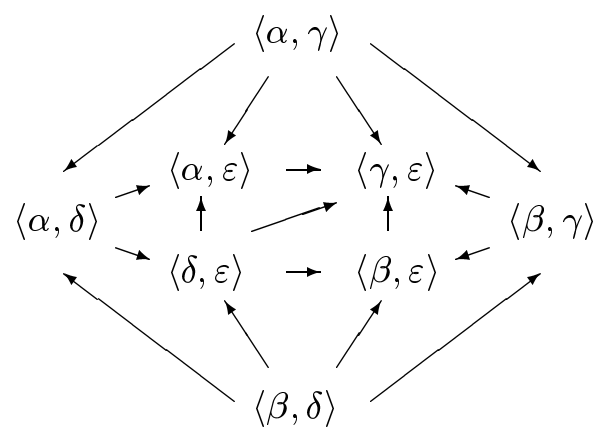

Fig. 4. Reduction of a square path

LemMa 11. Let $\alpha$ and $\beta$ be disjoint, non-separating meridian curves. Let $v_{1}$ and $v_{2}$ be vertices of $X$ connected by an edge and containing $\alpha$ and $\beta$ respectively (or $v_{1}=v_{2}$ contains both curves $\alpha$ and $\beta$ ). Let $v_{3}$ and $v_{4}$ be another pair of vertices connected by an edge and containing $\alpha$ and $\beta$ 
respectively. Then there exist an $\alpha$-segment connecting $v_{1}$ and $v_{3}$ and a $\beta$-segment connecting $v_{2}$ and $v_{4}$ such that the union of the two segments, together with edges connecting $v_{1}$ to $v_{2}$ and $v_{3}$ to $v_{4}$, forms a closed edge-path null-homotopic in $X$.

Proof. Suppose first that $\alpha \cup \beta$ does not separate $S$. When we cut $B$ along meridian disks bounded by $\alpha$ and $\beta$ we get a handlebody $B_{1}$ of genus $g-2$. We can choose $g-2$ curves out of the common curves of $v_{1}$ and $v_{2}$ which form a cut-system $u_{1}$ on $B_{1}$. We can also choose $g-2$ curves out of the common curves of $v_{3}$ and $v_{4}$ which form a cut-system $w_{1}$ on $B_{1}$.

We connect $u_{1}$ to $w_{1}$ by an edge-path in the cut-system complex of $B_{1}$. We add $\alpha$ and $\beta$ to all vertices of this edge-path. We get an $(\alpha, \beta)$-segment. The first vertex of this segment is connected by edges to $v_{1}$ and $v_{2}$ and forms a triangle (or is equal to $v_{1}$ if $v_{1}=v_{2}$ ). The last vertex is connected by edges to $v_{3}$ and $v_{4}$ and forms another triangle (see Figure 5 , right side). We can extend the $(\alpha, \beta)$-segment to an $\alpha$-segment from $v_{1}$ to $v_{3}$ and to a $\beta$-segment from $v_{2}$ to $v_{4}$. The union of the segments and the edges is equal to a sum of two triangles and a trivial path.

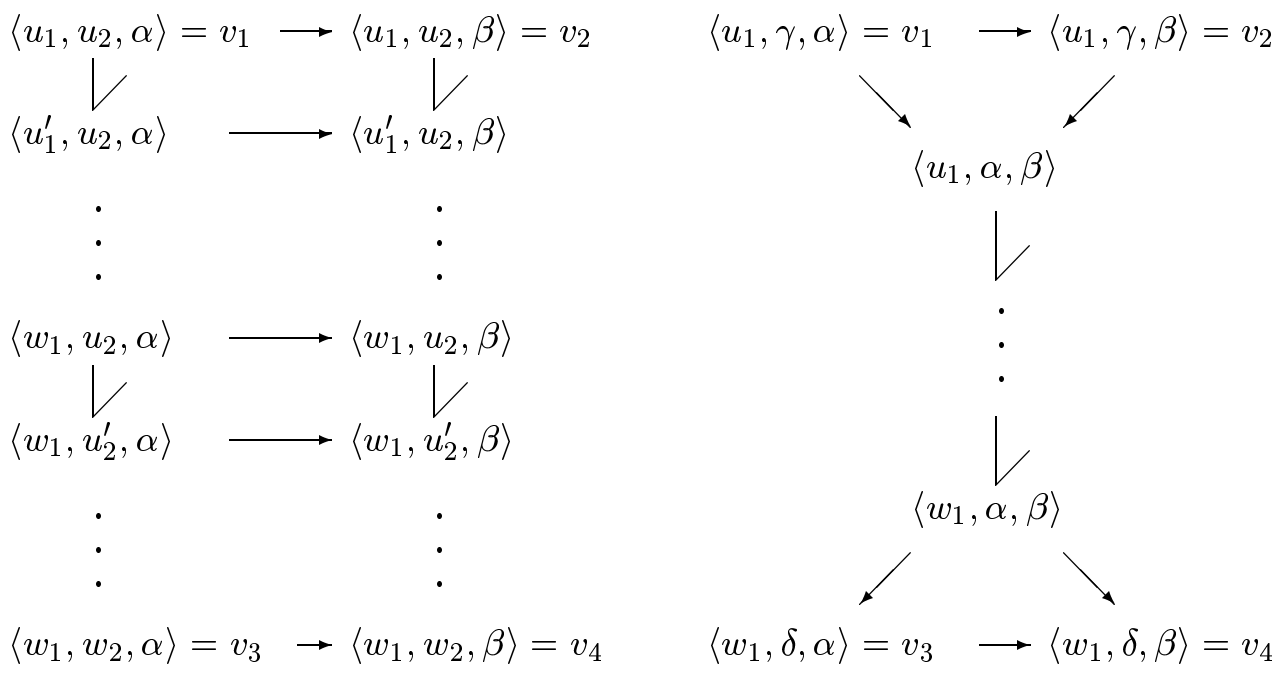

Fig. 5. Parallel segments

Suppose now that $\alpha \cup \beta$ separates $S$. When we cut $B$ along meridian disks bounded by $\alpha$ and $\beta$ we get two handlebodies $B_{1}$ and $B_{2}$ and the sum of the genera of $B_{1}$ and $B_{2}$ equals $g-1$. The remaining curves, common to $v_{1}$ and $v_{2}$, split into two families; one forms a cut-system $u_{1}$ on $B_{1}$ and the other forms a cut-system $u_{2}$ on $B_{2}$. Also the remaining curves in $v_{3}$ and $v_{4}$ 
form a cut-system $w_{1}$ on $B_{1}$ and a cut-system $w_{2}$ on $B_{2}$. We connect $u_{1}$ to $w_{1}$ by an edge-path in the cut-system complex of $B_{1}$. We add $\alpha$ and the curves of $u_{2}$ to all vertices of the edge-path getting an edge-path in $X$ (see Figure 5, left side).

Now we connect $u_{2}$ to $w_{2}$ by an edge-path in the cut-system complex of $B_{2}$ and add $\alpha$ and all curves of $w_{1}$ to all vertices of this edge-path. We get a new edge-path in $X$ which extends the first edge-path. Together we get an $\alpha$-segment connecting $v_{1}$ to $v_{3}$ in $X$. Now we replace $\alpha$ by $\beta$ in all vertices of this segment and get a $\beta$-segment connecting $v_{2}$ to $v_{4}$ in $X$. Corresponding vertices in the two segments are joined by an edge. The union of the segments together with the edges connecting $v_{1}$ to $v_{2}$ and $v_{3}$ to $v_{4}$ splits into squares which are null-homotopic by Lemma 10.

Lemma 12. A closed edge-path $\mathbf{p}$ of radius zero around a curve $\alpha$ of some vertex of $\mathbf{p}$ is null-homotopic in $X$.

Proof. We prove the lemma by induction on the number of segments having a common curve disjoint from $\alpha$. If all vertices contain $\alpha$ then we have a closed segment. In the other case consider a maximal segment containing $\alpha$. Let $u_{1}$ be its first vertex and let $v_{1}$ be its last vertex. Let $u_{2}$ be the next vertex of $\mathbf{p}$. The vertex $u_{2}$ contains a curve $\beta$ disjoint from $\alpha$, the common curve of the second segment. Let $v_{2}$ be the last vertex of the second segment. Suppose that there are only two segments. We are in the situation of Lemma 11 . We can connect the two pairs of vertices $v_{1}, u_{2}$ and $v_{2}, u_{1}$ by parallel segments and $\mathbf{p}$ splits into a sum of two closed segments and a path null-homotopic by Lemma 11 . So we may assume that there exists a third segment with a common curve $\gamma$ disjoint from $\alpha$ and $\beta$. Let $u_{3}$ be the vertex of $\mathbf{p}$ following $v_{2}$. It contains $\gamma$. We cut $B$ along meridian disks bounded by $\alpha, \beta, \gamma$.

If we get three components then the genus goes down by one. We choose a cut-system on each component, add them up and get $g-1$ curves. When we also add the curve $\alpha$ (respectively $\beta, \gamma$ ) we get a cut-system $w_{1}$ (respectively $\left.w_{2}, w_{3}\right)$ on $B$ and the cut-systems form a triangle. By Lemma 11 we can connect $w_{1}$ to $v_{1}$ by an $\alpha$-segment, $w_{2}$ to $u_{2}$ and $v_{2}$ by $\beta$-segments and $w_{3}$ to $u_{3}$ by a $\gamma$-segment. Figure 6 shows that $\mathbf{p}$ can be replaced by an edgepath which does not have a $\beta$-segment and the difference of the edge-paths decomposes into a sum of null-homotopic paths by Lemmas 9 and 11. If $\alpha \cup \beta$ does not separate $S$ we can find a vertex $w_{1}=w_{2}$ which contains both curves $\alpha$ and $\beta$ and is connected by an edge to a vertex $w_{3}$ containing $\gamma$. The case of $\alpha \cup \gamma$ or $\beta \cup \gamma$ non-separating is similar. If $\alpha \cup \beta \cup \gamma$ does not separate we can find a vertex $w_{1}=w_{2}=w_{3}$ containing all three curves. The argument using Figure 6 works in all cases. Lemma 12 follows by induction on the number of segments. 


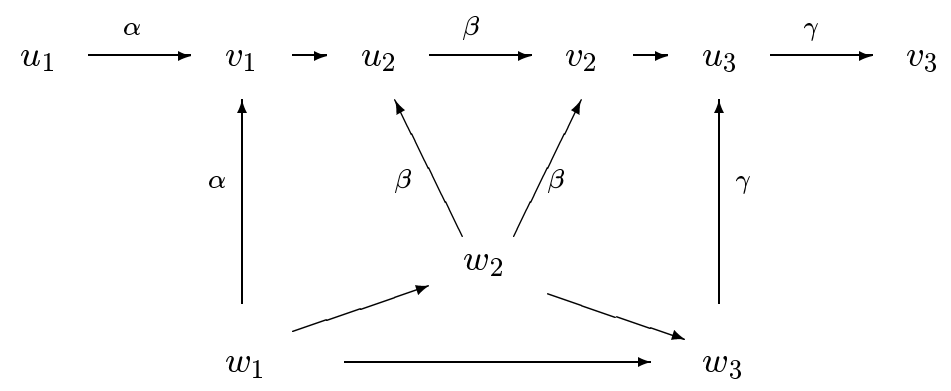

Fig. 6. Reduction of a path of radius 0

The next lemma will be used to make a short-cut from a vertex $u_{1}$ to $u_{2}$ in the situation of Figure 7.

Lemma 13. Let $\alpha, \beta, \gamma_{1}, \gamma_{2}$ be non-separating curves on $S$ such that $\left|\gamma_{1} \cap \gamma_{2}\right|=k,\left|\gamma_{1} \cap \alpha\right|<m,\left|\gamma_{2} \cap \alpha\right| \leq m$ and $\left|\gamma_{1} \cap \beta\right|=\left|\gamma_{2} \cap \beta\right|=0$. Let $u_{1}$ and $u_{2}$ be vertices of $X$ containing $\gamma_{1}$ and $\gamma_{2}$ respectively. Then there exists a path $\mathbf{q}$ connecting $u_{1}$ and $u_{2}$ such that all vertices of $\mathbf{q}$ have distance zero from $\beta$ and have distance less than $m$ from $\alpha$, except possibly for a final $\gamma_{2}$-segment of $\mathbf{q}$ which ends at $u_{2}$.

Proof. If $\left|\gamma_{1} \cap \gamma_{2}\right|=0$ then we can find vertices $w_{1}$ and $w_{2}$ connected by an edge in $X$ and containing $\gamma_{1}$ and $\gamma_{2}$ respectively (possibly one vertex contains both). We can connect $u_{1}$ to $w_{1}$ by a $\gamma_{1}$-segment and $w_{2}$ to $u_{2}$ by a $\gamma_{2}$-segment, by Lemma 6 , getting the required edge-path $\mathbf{q}$.

If $\left|\gamma_{1} \cap \gamma_{2}\right|=k>0$ then, by Lemma 5, we can find a non-separating meridian curve $\delta$ such that $|\delta \cap \beta|=0,|\delta \cap \alpha|<m,\left|\delta \cap \gamma_{1}\right|<k$, and $\left|\delta \cap \gamma_{2}\right|<k$. Let $w$ be a vertex containing $\delta$. By induction on $k$ we can find an edge-path $\mathbf{q}_{1}$ between $u_{1}$ and $w$ and an edge-path $\mathbf{q}_{2}$ between $w$ and $u_{2}$ such that both have radius zero around $\beta$, all vertices of $\mathbf{q}_{1}$ have distance less than $m$ from $\alpha$ and all vertices of $\mathbf{q}_{2}$ have distance less than $m$ from $\alpha$, except possibly for the final $\gamma_{2}$-segment which ends at $u_{2}$. Together they form the required edge-path $\mathbf{q}$.

Proof of Theorem 1. $X$ is connected by Proposition 8. We want to prove that every closed edge-path in $X$ is null-homotopic. Suppose that a closed path $\mathbf{p}$ has radius $m$ around some curve $\alpha$. If $m=0$ then $\mathbf{p}$ is null-homotpic by Lemma 12. We continue by induction on $m$. For fixed $m$ we prove the result by induction on the number of segments of $\mathbf{p}$ which have a common curve at distance $m$ from $\alpha$.

Let $v_{0}$ be a vertex of $\mathbf{p}$ containing $\alpha$. We say that $\mathbf{p}$ begins at $v_{0}$. Let $v_{1}$ be the first vertex of $\mathbf{p}$ which has distance $m$ from $\alpha$. Let $\mathbf{q}_{0}$ be the maximal segment of $\mathbf{p}$ which starts at $v_{1}$, contains some fixed curve $\beta$ satisfying $|\beta \cap \alpha|=m$ and such that no vertex of $\mathbf{q}_{0}$ contains a curve $\beta^{\prime}$ 
satisfying $\left|\beta^{\prime} \cap \alpha\right|<m$. Let $v_{2}$ be the last vertex of $\mathbf{q}_{0}$. Let $u_{1}$ be the vertex of $\mathbf{p}$ preceding $v_{1}$ and let $u_{2}$ be the vertex of $\mathbf{p}$ following $v_{2}$ (see Figure 7 ). The vertex $u_{1}$ contains a curve $\gamma_{1}$ such that $\left|\gamma_{1} \cap \alpha\right|<m$. The vertex $u_{2}$ contains a curve $\gamma_{2}$ such that $\left|\gamma_{2} \cap \alpha\right|<m$ or $\left|\gamma_{2} \cap \alpha\right|=m$ and $\gamma_{2}$ is the common curve of the next segment. Since the curves in the neighbouring vertices are disjoint we have $\left|\beta \cap \gamma_{1}\right|=\left|\beta \cap \gamma_{2}\right|=0$.

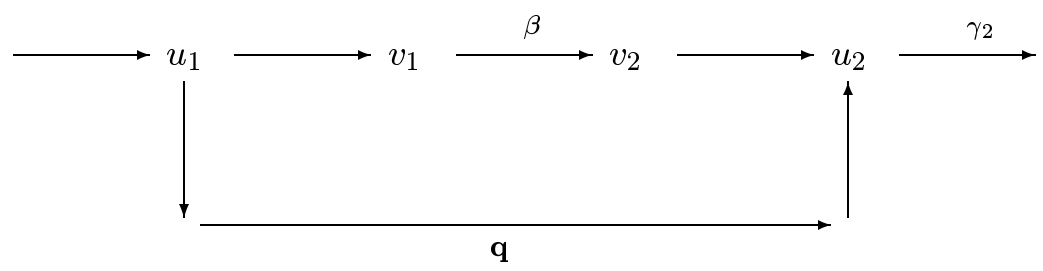

Fig. 7. Reduction of a path of radius $m$

We can now replace the part of $\mathbf{p}$ from $u_{1}$ to $u_{2}$ by a path $\mathbf{q}$ from Lemma 13 (see Figure 7 ). The new path $\mathbf{p}_{1}$ differs from $\mathbf{p}$ by a closed path of radius zero around $\beta$, so $\mathbf{p}_{1}$ is homotopic to $\mathbf{p}$, and it has no $\beta$-segment (although its $\gamma_{2}$-segment may be longer than in $\mathbf{p}$ ). The number of segments in $\mathbf{p}_{1}$ at distance $m$ from $\alpha$ is smaller than in $\mathbf{p}$. Theorem 1 follows by induction.

2. A presentation of $\mathcal{M}_{g}$. In this section we establish a presentation of the mapping class group of a handlebody. We denote by $B=B_{g}$ a handlebody of genus $g$ represented in Figure 1. We denote by $S$ the boundary of $B$, by $\mathcal{M}$ the mapping class group of $B$ and by $X$ the cut-system complex of $B$.

For $g=1$ one can compute the mapping class group directly:

Theorem 14. The mapping class group $\mathcal{M}_{1}$ of a solid torus $B$ is isomorphic to $\mathbb{Z} \oplus \mathbb{Z}_{2}$.

Proof. If $g=1$ then the curve $\alpha_{1}$ in Figure 1 is the only meridian curve on the boundary $S$ of $B$, up to isotopy. A homeomorphism $h$ of $B$ either leaves the isotopy class of $\alpha_{1}$ invariant or, up to isotopy, $h$ fixes $\alpha_{1}$ setwise and reverses its orientation, so that $h^{2}$ leaves the isotopy class of $\alpha_{1}$ invariant. The $z$-axis in Figure 1 is shown for $g$ even. For odd genus the $z$-axis passes through the middle handle. In the case of genus one the rotation $z$ through 180 degrees around the $z$-axis reverses the orientation of $\alpha_{1}$, and $z^{2}=1$. So for every $h \in \mathcal{M}_{1}$ either $h$ or $z h$ preserves the isotopy class of $\alpha_{1}$. If a homeomorphism $h$ preserves the isotopy class of $\alpha_{1}$ then it is isotopic to a homeomorphism fixed on $\alpha_{1}$. It induces a homeomorphism of the annulus $S-\alpha_{1}$ and thus it is a power of the Dehn twist $a_{1}$ with respect to the curve $\alpha_{1}$ (see Definition 1 below). The elements $a_{1}$ and $z$ of $\mathcal{M}_{1}$ commute. The theorem follows. 
From now on we assume that $g>1$.

To shorten some formulas we adopt the following notation for conjugation: $a * b=a b a^{-1}$. As usual, $[a, b]=a b a^{-1} b^{-1}$.

REMARK 1. Some proofs of relations between homeomorphisms of a surface are left to the reader. The general idea of the proof is as follows. We split the surface into a union of disks by a finite number of curves (and arcs with the end-points on the boundary if the surface has a boundary). We prove that the given product of homeomorphisms takes each curve (respectively arc) onto an isotopic curve (arc), preserving some fixed orientation of the curve (arc). Then the product is isotopic to a homeomorphism pointwise fixed on each curve and arc. But a homeomorphism of a disk fixed on its boundary is isotopic to the identity homeomorphism, relative to the boundary, by the Lemma of Alexander. Thus the given product of homeomorphisms is isotopic to the identity.

We start with some properties of homeomorphisms of the surface $S$. Dehn proved in [4] that every homeomorphism of $S$ is isotopic to a product of twists of the following type.

Definition 1. A (positive) Dehn twist with respect to a simple closed curve $\alpha$ on an oriented surface $S$ is the isotopy class of a homeomorphism $h$ of $S$, supported in a regular neighbourhood $N$ of $\alpha$ (an annulus), obtained as follows: we cut $S$ open along $\alpha$, rotate one side of the cut by 360 degrees to the right (this makes sense on an oriented surface) and then glue the surface back together, damping out the rotation to the identity at the boundary of $N$. The Dehn twist (or simply twist) with respect to $\alpha$ will be denoted by $T_{\alpha}$.

Lemma 15. Let $\alpha$ be a curve on $S$, let $h$ be a homeomorphism and let $\alpha^{\prime}=h(\alpha)$. Then $T_{\alpha^{\prime}}=h T_{\alpha} h^{-1}$.

Proof. Since $h$ maps $\alpha$ to $\alpha^{\prime}$ we may assume that (up to isotopy) it also maps a neighbourhood $N$ of $\alpha$ to a neighbourhood $N^{\prime}$ of $\alpha^{\prime}$. The homeomorphism $h^{-1}$ takes $N^{\prime}$ to $N$, then $T_{\alpha}$ maps $N$ to $N$, twisting along $\alpha$, and $h$ takes $N$ back to $N^{\prime}$. Since $T_{\alpha}$ is supported in $N$, the composite map is supported in $N^{\prime}$ and it is a Dehn twist about $\alpha^{\prime}$.

Lemma 16. Let $\gamma_{1}, \ldots, \gamma_{k}$ be a chain of curves, i.e. the consecutive curves intersect once and non-consecutive curves are disjoint. Let $N$ denote a regular neighbourhood of the union of these curves. Let $c_{i}$ denote the twist along $\gamma_{i}$. Then the following relations hold:

(i) The "commutativity relation": $c_{1} c_{3}=c_{3} c_{1}$.

(ii) The "braid relation": $c_{1} c_{2}\left(\gamma_{1}\right)=\gamma_{2}, c_{1} c_{2} c_{1}=c_{2} c_{1} c_{2}$. 
(iii) The "chain relation": If $k$ is odd then $N$ has two boundary components, $\partial_{1}$ and $\partial_{2}$, and $\left(c_{1} \ldots c_{k}\right)^{k+1}=T_{\partial_{1}} T_{\partial_{2}}$. If $k$ is even then $N$ has one boundary component $\partial_{1}$ and $\left(c_{1} \ldots c_{k}\right)^{2 k+2}=T_{\partial_{1}}$.

(iv) $\left(c_{2} c_{1} c_{3} c_{2}\right)\left(c_{4} c_{3} c_{5} c_{4}\right)\left(c_{2} c_{1} c_{3} c_{2}\right)=\left(c_{4} c_{5} c_{3} c_{4}\right)\left(c_{2} c_{1} c_{3} c_{2}\right)\left(c_{4} c_{3} c_{5} c_{4}\right)$.

Proof. Relation (i) is obvious. It follows immediately from the definition of Dehn twist that $c_{2}\left(\gamma_{1}\right)=c_{1}^{-1}\left(\gamma_{2}\right)$. Both statements of (ii) follow from this and from Lemma 15. Relation (iii) is a little more complicated, but we shall only use it for $k=2,3$, for which it is well known (see [14]).

We prove (iv). The proof involves a long sequence of braid transformations (relations (i) and (ii)):

$$
\begin{aligned}
& \left(c_{2} c_{1} c_{3} c_{2}\right)\left(c_{4} c_{3} c_{5} c_{4}\right)\left(c_{2} c_{1} c_{3} c_{2}\right)=c_{2} c_{3} c_{1} c_{2} c_{4} c_{5} c_{3} c_{4} c_{2} c_{3} c_{1} c_{2} \\
& =c_{2} c_{3} c_{4} c_{1} c_{5} c_{2} c_{3} c_{2} c_{4} c_{3} c_{1} c_{2}=c_{2} c_{3} c_{4} c_{3} c_{1} c_{5} c_{2} c_{3} c_{4} c_{3} c_{1} c_{2} \\
& =c_{4} c_{2} c_{3} c_{4} c_{1} c_{5} c_{2} c_{4} c_{3} c_{1} c_{2} c_{4}=c_{4} c_{2} c_{3} c_{1} c_{4} c_{5} c_{4} c_{2} c_{3} c_{1} c_{2} c_{4} \\
& =c_{4} c_{5} c_{2} c_{3} c_{1} c_{4} c_{2} c_{3} c_{1} c_{2} c_{5} c_{4}=c_{4} c_{5} c_{2} c_{3} c_{1} c_{2} c_{1} c_{4} c_{3} c_{2} c_{5} c_{4} \\
& =c_{4} c_{5} c_{3} c_{2} c_{3} c_{4} c_{1} c_{2} c_{3} c_{2} c_{5} c_{4}=c_{4} c_{5} c_{3} c_{2} c_{3} c_{4} c_{3} c_{1} c_{2} c_{3} c_{5} c_{4} \\
& =c_{4} c_{5} c_{3} c_{4} c_{2} c_{3} c_{4} c_{1} c_{2} c_{3} c_{5} c_{4}=\left(c_{4} c_{5} c_{3} c_{4}\right)\left(c_{2} c_{1} c_{3} c_{2}\right)\left(c_{4} c_{3} c_{5} c_{4}\right)
\end{aligned}
$$

LEMMA 17. Let $U$ be a disk with outer boundary $\partial$ and with $n$ inner holes bounded by curves $\partial_{1}, \ldots, \partial_{n}$. For $1 \leq i<j \leq n$ let $\alpha_{i, j}$ be the simple closed curve in $U$ shown in Figure 8, separating two holes $\partial_{i}$ and $\partial_{j}$ from the other holes. Let $d$ be the twist along $\partial$, let $d_{i}$ be the twist along $\partial_{i}$, and let $a_{i, j}$ be the twist along $\alpha_{i, j}$. Then

$d=\left(a_{1,2} a_{1,3} \ldots a_{1, n} a_{2,3} \ldots a_{2, n} a_{3,4} \ldots a_{n-2, n-1} a_{n-2, n} a_{n-1, n}\right)\left(d_{1} \ldots d_{n}\right)^{2-n}$.

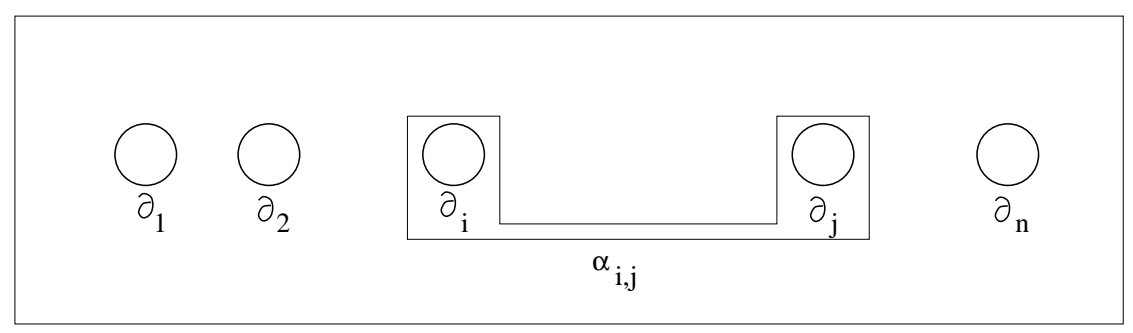

Fig. 8. Disk with holes and the curves $\alpha_{i, j}$

Proof. If we allow isotopies which rotate the curves $\partial_{i}$ then the group of the isotopy classes of homeomorphisms of $U$ is isomorphic to the group of the isotopy classes of homeomorphisms which preserve punctures of a disk with $n$ punctures. This is the classical pure braid group on $n$ strings. The elements $a_{i, j}$ represent the standard generators of the pure braid group and 
$d$ is equal to the generator $\Delta^{2}$ of the centre. Therefore, modulo the twists $d_{i}$,

$$
d=\left(a_{1,2} a_{1,3} \ldots a_{1, n} a_{2,3} \ldots a_{2, n} a_{3,4} \ldots a_{n-2, n-1} a_{n-2, n} a_{n-1, n}\right) .
$$

The factor corresponding to the twists $d_{i}$ can be checked by induction (by drawing many pictures).

The special case of the above lemma, for $n=3$, was observed by Dennis Johnson [10] and called a lantern relation.

We now fix some curves on $S$. The handlebody $B$ consists of $g$ handles attached to a 3-ball. For $i=1, \ldots, g$ and $j=1, \ldots, g-1$ we fix curves $\alpha_{i}$, $\beta_{i}, \varepsilon_{j}$ (see Figure 1). The curve $\alpha_{i}$ is a meridian curve across the $i$ th handle, $\beta_{i}$ is a curve along the $i$ th handle and $\varepsilon_{i}$ runs along the $i$ th handle and the $(i+1)$ st handle. The curves $\alpha_{1}, \ldots, \alpha_{g}$ form a cut-system.

We denote by $I_{0}$ the set of indices $\{-g, 1-g, \ldots,-1,1,2, \ldots, g\}$.

When we cut $S$ open along the curves $\alpha_{1}, \ldots, \alpha_{g}$ we get a sphere $S_{0}$ with $2 g$ holes bounded by curves $\partial_{i}, i \in I_{0}$, where $\partial_{i}$ and $\partial_{-i}$ correspond to the same curve $\alpha_{i}$ on $S$ (see Figure 10). The glueing back map identifies $\partial_{i}$ with $\partial_{-i}$ according to the reflection with respect to the $x$-axis. Curves on $S$ can be represented on $S_{0}$. If a curve on $S$ meets some curves $\alpha_{i}$ then it is represented on $S_{0}$ by a disjoint union of several arcs. In particular $\varepsilon_{i}$ is represented by two arcs joining $\partial_{-i}$ to $\partial_{-i-1}$ and $\partial_{i}$ to $\partial_{i+1}$. We denote by $\delta_{i, j}, i<j \in I_{0}$, the curves represented in Figures 1 and 10 . The curve $\delta_{i, j}$ separates the holes $\partial_{i}$ and $\partial_{j}$ from the other holes on $S_{0}$.

DeFinition 2. For each subset $I=\left\{i_{1}, \ldots, i_{n}\right\}$ of $I_{0}$ with $i_{1}<\ldots<i_{n}$ we define a standard curve $\delta_{I}$ in $S_{0}$ (see Figure 9), which separates the holes $\partial_{i_{1}}, \ldots, \partial_{i_{n}}$ from the other holes in $S_{0}$. We see that if $i, j \in I$ then $\delta_{i, j}$ is contained inside $\delta_{I}$. If $I$ contains just two indices $i, j$ then $\delta_{I}=\delta_{i, j}$, as in Figure 10. For $i \leq j \in I_{0}$ and $I=\left\{k \in I_{0} \mid i \leq k \leq j\right\}$ we define a curve $\gamma_{i, j}=\delta_{I}$.

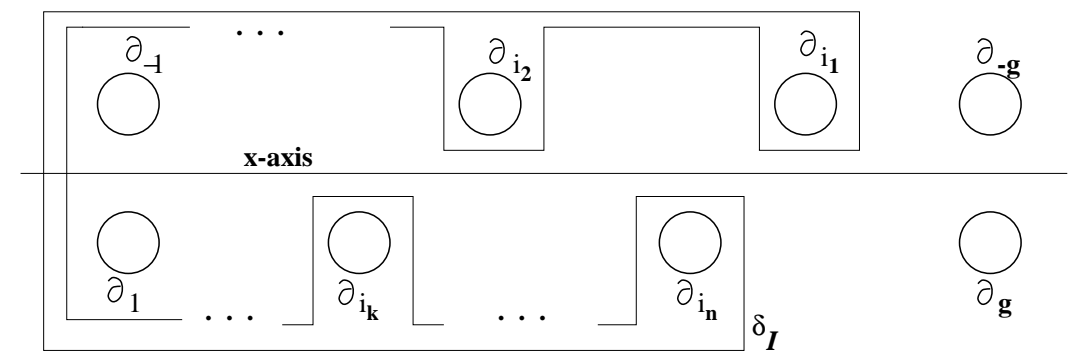

Fig. 9. Standard curve $\delta_{I}$ on $S_{0}$ for $I=\left\{i_{1}, \ldots, i_{n}\right\}$

We now fix some elements of $\mathcal{M}$. A homeomorphism $h$ of $S$ represents an element in $\mathcal{M}$ if it takes curves of some cut-system $\left\langle\gamma_{1}, \ldots, \gamma_{g}\right\rangle$ to curves 


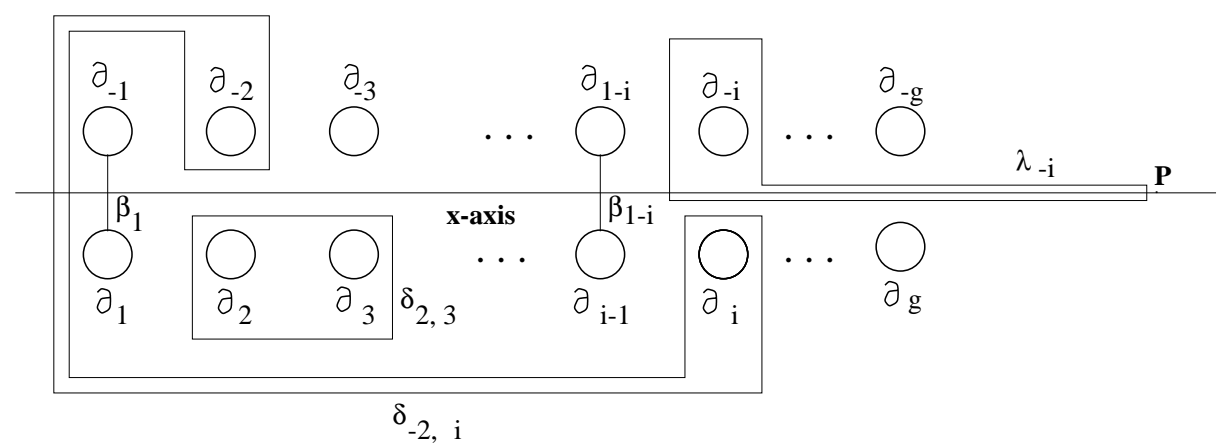

Fig. 10. Curves $\delta_{i, j}$ and $\lambda_{k}$ on $S_{0}$

of a cut-system. Indeed, we can extend $h$ to disjoint meridian disks bounded by $\gamma_{1}, \ldots, \gamma_{g}$. When we cut $B$ open along the meridian disks we get a ball and $h$ can be extended from the boundary of the ball to its interior. A twist with respect to a curve $\alpha$ belongs to $\mathcal{M}$ if and only if $\alpha$ is a meridian curve. A curve which is disjoint from all curves of some cut-system is a meridian curve.

Definition 3. We denote by $a_{i}, b_{i}, e_{j}$ the Dehn twists along the curves $\alpha_{i}, \beta_{i}, \varepsilon_{j}$ respectively and we denote by $d_{1,2}$ the Dehn twist with respect to $\delta_{1,2}$. For $i \in I_{0}$ we denote by $d_{i}$ the twist of $S_{0}$ along the curve $\partial_{i}$. It can be identified with the twist $a_{|i|}$ of $S$. We fix the following elements of $\mathcal{M}$ :

$$
\begin{aligned}
s & =b_{1} a_{1} a_{1} b_{1}, \\
t_{i} & =e_{i} a_{i} a_{i+1} e_{i} \quad \text { for } i=1, \ldots, g-1 .
\end{aligned}
$$

For $i<j \in I_{0}$ we let

$$
\begin{aligned}
d_{i, j} & =\left(t_{i-1} t_{i-2} \ldots t_{1} t_{j-1} t_{j-2} \ldots t_{2}\right) * d_{1,2} \quad \text { if } i>0, \\
d_{i, j} & =\left(t_{-i-1}^{-1} t_{-i-2}^{-1} \ldots t_{1}^{-1} s^{-1} t_{j-1} t_{j-2} \ldots t_{2}\right) * d_{1,2} \quad \text { if } i<0 \text { and } i+j>0, \\
d_{i, j} & =\left(t_{-i-1}^{-1} t_{-i-2}^{-1} \ldots t_{1}^{-1} s^{-1} t_{j} t_{j-1} \ldots t_{2}\right) * d_{1,2} \\
d_{i, j} & =\left(t_{-j-1}^{-1} t_{-j-2}^{-1} \ldots t_{1}^{-1} t_{-i-1}^{-1} t_{-i-2}^{-1} \ldots t_{2}^{-1} s^{-1} t_{1}^{-1} s^{-1}\right) * d_{1,2} \quad \text { if } j<0, \\
d_{i, j}= & \left(t_{j-1}^{-1} d_{j-1, j} t_{j-2}^{-1} d_{j-2, j-1} \ldots t_{1}^{-1} d_{1,2}\right) *\left(s^{2} a^{4}\right) \quad \text { if } i+j=0, \\
d_{I} & =\left(d_{i_{1}, i_{2}} d_{i_{1}, i_{3}} \ldots d_{i_{1}, i_{n}} d_{i_{2}, i_{3}} \ldots d_{i_{2}, i_{n}} d_{i_{3}, i_{4}} \ldots d_{i_{n-1}, i_{n}}\right)\left(a_{i_{1}} \ldots a_{i_{n}}\right)^{2-n}
\end{aligned}
$$

where $I=\left\{i_{1}, \ldots, i_{n}\right\} \subset I_{0}, i_{1}<\ldots<i_{n}$,

$c_{i, j}=d_{I}, \quad$ where $i \leq j \in I_{0}$ and $I=\left\{k \in I_{0} \mid i \leq k \leq j\right\}$,

$r_{i, j}=b_{j} a_{j} c_{i, j} b_{j}, \quad$ where $i \leq j \in I_{0}$ and $I=\left\{k \in I_{0} \mid i \leq k \leq j\right\}$,

$k_{j}=a_{j} a_{j+1} t_{j} d_{j, j+1}^{-1} \quad$ for $j=1, \ldots, g-1$,

$s_{1}=s, \quad$ and $s_{j}=\left(k_{j-1} k_{j-2} \ldots k_{1}\right) * s_{1} \quad$ for $j=2, \ldots, g$, 


$$
\begin{aligned}
z & =a_{1} a_{2} \ldots a_{g} s t_{1} t_{2} \ldots t_{g-1} s t_{1} \ldots t_{g-2} s \ldots s t_{1} s d_{I} \\
z_{j} & =\left(k_{j-1} k_{j-2} \ldots k_{g+1-j}\right) * z \quad \text { if } j>g / 2 .
\end{aligned} \quad \text { where } I=\{1, \ldots, g\},
$$

Most of the products in the above definition represent very simple elements of $\mathcal{M}$ and we now explain their meaning. We first define special homeomorphisms of $S_{0}$.

Definition 4. A half-twist $h_{i, j}$ along a curve $\delta_{i, j}$ is the isotopy class (on $S_{0}$ relative to its boundary) of a homeomorphism of $S_{0}$ which is fixed outside $\delta_{i, j}$ and is equal to a counterclockwise "rotation" through 180 degrees inside $\delta_{i, j}$. In particular $h_{i, j}$ switches the two holes $\partial_{i}$ and $\partial_{j}$ inside $\delta_{i, j}$ so it is not fixed on the boundary of $S_{0}$, but $h_{i, j}^{2}$ is fixed on the boundary of $S_{0}$ and is isotopic to the full Dehn twist along $\delta_{i, j}$.

One can check that $t_{i}=h_{i, i+1} h_{-i-1,-i} a_{i}^{-1} a_{i+1}^{-1}$ and $s=h_{-1,1} a_{1}^{-2}$. So $t_{i}$ switches the holes $\partial_{i}$ and $\partial_{i+1}$ rotating the interior of $\delta_{i, i+1}$ counterclockwise and it switches the holes $\partial_{-i-1}$ and $\partial_{-i}$ rotating the interior of $\delta_{-i-1,-i}$ counterclockwise, and $s$ switches the holes $\partial_{1}$ and $\partial_{-1}$ rotating the interior of $\delta_{-1,1}$ counterclockwise. This interpretation of $t_{i}$ and $s$ is very useful in the investigation of their action on curves in $S_{0}$.

It follows from Lemma 16 that $s\left(\alpha_{i}\right)=\alpha_{i}$ for $i=1, \ldots, g, t_{i}\left(\alpha_{i}\right)=\alpha_{i+1}$, $t_{i}\left(\alpha_{i+1}\right)=\alpha_{i}$, and $t_{i}\left(\alpha_{j}\right)=\alpha_{j}$ for $j \neq i, i+1$. Thus the elements $a_{i}, s$, and $t_{i}$ belong to $\mathcal{M}$.

We now consider the products $d_{i, j}$. For $i \neq-j$ we start with $\delta_{1,2}$ and apply consecutive factors $t_{i}$ and $s$, one at a time. We check that the result is $\delta_{i, j}$ so $d_{i, j}$ is the twist along $\delta_{i, j}$, by Lemma 15 . For $d_{-1,1}$ we use Lemma 16(iii). The curve $\delta_{-1,1}$ is the boundary of a regular neighbourhood of $\alpha_{1} \cup \beta_{1}$ and $\left(a_{1} b_{1}\right)^{6}=s^{2} a_{1}^{4}$ by Lemma 16(i), (ii), therefore $d_{-1,1}=\left(a_{1} b_{1}\right)^{6}$ is the twist along $\delta_{-1,1}$ by Lemma 16(iii). Now we apply a suitable product of $t_{i}$ 's and $d_{i, i+1}$ 's to $\delta_{-1,1}$ and get $\delta_{-i, i}$. Thus $d_{i, j}$ is the twist along $\delta_{i, j}$ for all $i, j$.

It follows from Lemma 17 that $d_{I}$ is equal to the twist along $\delta_{I}$. In particular $c_{i, j}$ is the twist along the curve $\gamma_{i, j}$.

Now it follows from Lemma 16 that $r_{i, j}\left(\alpha_{j}\right)=\gamma_{i, j}$. Also the curves $\alpha_{1}, \ldots$ $\ldots, \alpha_{j-1}, \gamma_{i, j}, \alpha_{j+1}, \ldots, \alpha_{g}$ form a cut-system. Thus $r_{i, j}$ belongs to $\mathcal{M}$.

One can check, by drawing pictures, that $k_{j}\left(\alpha_{j}\right)=\alpha_{j+1}$ and $k_{j}\left(\beta_{j}\right)=$ $\beta_{j+1}$, hence $s_{j}=b_{j} a_{j}^{2} b_{j}$ is essentially the half-twist along a curve surrounding the holes $\partial_{j}$ and $\partial_{-j}$ (see Definition 4 and the interpretation of $s$ ).

We may assume that the handlebody $B$ has a rotational symmetry with respect to the $z$-axis (the vertical axis in Figure 1), so the 180 degrees rotation around the $z$-axis takes the $B$ onto itself and takes the $i$ th handle onto the $(g+1-i)$ th handle. This rotation induces the rotation of $S_{0}$ through 180 degrees around the origin, which takes $\partial_{i}$ onto $\partial_{i-g-1}$ and $\partial_{-i}$ onto $\partial_{g+1-i}$ for $i=1, \ldots, g$. We now check that the element $z$ from 
Definition 3 represents the rotation. Let $z^{\prime}$ be the rotation. Consider loops around the holes $\partial_{i}, i<0$, which descend from above, from the common base point at $\infty$, and are disjoint outside $\infty$. Consider also loops around the holes $\partial_{i}, i>0$, which ascend from below, from the common base point at $\infty$, and are disjoint outside $\infty$. It is easy to check, using Definition 4 , that $z$ and $z^{\prime}$ act in the same way on these loops. Since the complement of these loops is a union of a disk and of annuli around $\partial_{i}$ 's, it follows that $z$ and $z^{\prime}$ may only differ by a product of $a_{i}$ 's. It is easy to check that $z\left(\beta_{1}\right)=z^{\prime}\left(\beta_{1}\right)=\beta_{g}$. Since $a_{g}\left(\beta_{g}\right) \neq \beta_{g}$ the power of $a_{g}$ in $z$ must be correct. Drawing some more pictures one can check that $z\left(\beta_{j}\right)=z^{\prime}\left(\beta_{j}\right)=\beta_{g+1-j}$, therefore the power of $a_{g+1-j}$ in $z$ must be correct for $j=1, \ldots, g$. So $z=z^{\prime}$ is the rotation. From this one can check that $z_{j}$ leaves the curves $\alpha_{j}$ and $\gamma_{j-g, j}$ invariant.

We can now state our main result, a finite presentation for $\mathcal{M}$ :

THEOREM 18. The mapping class group $\mathcal{M}_{g}$ of a handlebody of genus $g$ admits the following presentation:

The set of generators consists of $a_{1}, \ldots, a_{g}, d_{1,2}, s, t_{1}, \ldots, t_{g-1}$ and $r_{i, j}$ for $i<j \in I_{0}$ and $i=1$ or $(i<0, i+j>0, j-i \leq g)$.

The set of defining relations is:

(P1) $\left[a_{i}, a_{j}\right]=1$ and $\left[a_{i}, d_{j, k}\right]=1$ for all $i, j, k \in I_{0}$.

(P2) Pure braid relations

(a) $d_{r, s}^{-1} * d_{i, j}=d_{i, j}$ if $r<s<i<j \in I_{0}$ or $i<r<s<j \in I_{0}$,

(b) $d_{r, i}^{-1} * d_{i, j}=d_{r, j} * d_{i, j}$ if $r<i<j \in I_{0}$,

(c) $d_{i, s}^{-1} * d_{i, j}=\left(d_{i, j} d_{s, j}\right) * d_{i, j}$ if $i<s<j \in I_{0}$,

(d) $d_{r, s}^{-1} * d_{i, j}=\left[d_{r, j}, d_{s, j}\right] * d_{i, j}$ if $r<i<s<j \in I_{0}$.

(P3) $d_{I_{0}}=1$.

(P4) $d_{I_{k}}=a_{|k|}$ for every $k \in I_{0}$ where $I_{k}=I_{0}-\{k\}$.

(P5) $t_{i} t_{i+1} t_{i}=t_{i+1} t_{i} t_{i+1}$ for $i=1, \ldots, g-2$ and $\left[t_{i}, t_{j}\right]=1$ if $1 \leq i<$ $j-1<g-1$.

(P6) $s^{2}=d_{-1,1} a^{-4}$ and $t_{i}^{2}=d_{i, i+1} d_{-i-1,-i} a_{i}^{-2} a_{i+1}^{-2}$ for $i=1, \ldots, g-1$.

(P7) $\left[s, a_{i}\right]=1$ for $i=1, \ldots, g, t_{i} * a_{i}=a_{i+1}$ for $i=1, \ldots, g-1$, $\left[a_{i}, t_{j}\right]=1$ for $j \neq i, i-1$ and $\left[t_{i}, s\right]=1$ for $i=2, \ldots, g-1$.

(P8) $\left[s, d_{2,3}\right]=1,\left[s, d_{-2,2}\right]=1, s t_{1} s t_{1}=t_{1} s t_{1} s$, and $\left[t_{i}, d_{1,2}\right]=1$ for $i \neq 2$.

(P9) If $i<j \in I_{0}$ and $i=1$ or $(i<0, i+j>0, j-i \leq g)$ then $r_{i, j}^{2}=s_{j} c_{i, j} s_{j} c_{i, j}^{-1}$.

(P10) If $i<j \in I_{0}$ and $i=1$ or $(i<0, i+j>0, j-i \leq g)$ then

(a) $r_{i, j} * a_{j}=c_{i, j}$ and $\left[r_{i, j}, a_{k}\right]=1$ for $k \neq j$,

(b) $\left[r_{i, j}, t_{k}\right]=1$ if $k<|i|$ or $|i|<k<j-1$ or $k>j$ or $k=i=$ $1<j-1$

(c) $\left[r_{i, j}, s_{k}\right]=1$ if $k<|i|$ or $k>j$ or $k=-i$, 
(d) $\left[r_{i, j}, d_{k, m}\right]=1$ if $k, m \in\{i, \ldots, j-1\}$ or $k, m \notin\{-j, i, i+$ $1, \ldots, j\}$,

(e) $\left[r_{i, j}, z_{j}\right]=1$ if $j=g$ or $j-i=g$,

(f) $r_{i, j} * d_{i, j}=d_{J}$ where $J=\left\{k \in I_{0}: i<k \leq j\right\}$,

(g) $r_{1, j} * d_{-j, 1-j}=\left(t_{j-2} t_{j-3} \ldots t_{1}\right) * c_{-1, j}$,

(h) if $i<0$ and $j+i>1$ then $r_{i, j} * d_{-j, 1-j}=\left(t_{j-2} t_{j-3} \ldots t_{1-i}\right) *$ $c_{i-1, j}$

(k) if $j<g$ then $r_{i, j}^{-1} * d_{-j-1,-j}=s_{j+1}^{-1} * c_{i, j+1}$.

(P11) If $i<j \in I_{0}$ and $i=1$ or $(i<0, i+j>0, j-i \leq g)$ then $r_{i, j} * t_{j-1}=t_{j-1}^{-1} * r_{i, j}$.

(P12) For $g \geq j>2$ and $i=1$ or $(i<0, i+j>0, j-i \leq g)$

$$
\begin{aligned}
& r_{1, j}=s_{j} c_{1, j} s_{j} c_{1, j}^{-1} k_{j-1} a_{j} c_{1, j-2} t_{j-1} c_{1, j-1}^{-1} t_{j-1}^{-1} r_{1, j-1}^{-1} s_{j-1} h_{2} r_{1,2}^{-1} h_{2}^{-1} k_{j-1}^{-1} \text { with } \\
& h_{2}=k_{j-1}^{-1} t_{j-2}^{-1} t_{j-3}^{-1} \ldots t_{1}^{-1} k_{j-1} k_{j-2} \ldots k_{2}, \\
& r_{-1, j}=h_{3} r_{1,2}^{-1} h_{3}^{-1} s_{j} r_{1, j}^{-1} c_{-1, j-1}^{-1} c_{1, j-1} a_{1} s_{j} c_{-1, j} s_{j} c_{-1, j}^{-1} \quad \text { with } \\
& \quad h_{3}=s_{1} k_{j-1} k_{j-2} \ldots k_{2}, \\
& r_{i, j}=h_{3} r_{1,2}^{-1} h_{3}^{-1} s_{j} r_{i+1, j}^{-1} c_{i, j-1}^{-1} c_{i+1, j-1} a_{-i} s_{j} c_{i, j} s_{j} c_{i, j}^{-1} \quad \text { for } i<-1 \text { with } \\
& \quad h_{3}=s_{-i} t_{-1-i}^{-1} t_{-2-i}^{-1} \ldots t_{1}^{-1} k_{j-1} k_{j-2} \ldots k_{3} k_{2} .
\end{aligned}
$$

Although Theorem 18 is very long and complicated it has some simple and interesting consequences.

TheOrem 19. For $g>1$ the mapping class group $\mathcal{M}_{g}$ of a handlebody $B$ of genus $g$ is generated by five elements: $a_{1}, s_{1}, r_{1,2}, t_{1}$ and $u=t_{1} \ldots t_{g-1}$.

P roof. Let $G$ be the subgroup of $\mathcal{M}_{g}$ generated by $a_{1}, s_{1}, r_{1,2}, t_{1}, u$. We have to prove that $G$ contains the generators $a_{i}, s, d_{1,2}, t_{j}$ and $r_{i, j}$ of $\mathcal{M}$. We have $u * t_{i}=t_{i+1}$ by (P5), so $t_{j} \in G$ for all $j$. Now $t_{i} * a_{i}=a_{i+1}$ by (P7), so $a_{i} \in G$ for all $i$. By (P10) we have $r_{1,2} * a_{2}=d_{1,2} \in G$. Now all elements $d_{i, j}, s_{j}, k_{j}$ belong to $G$, by Definition 3 . Finally, $r_{i, j} \in G$ by (P12) and induction.

Theorem 20. The abelianized mapping class group $A=\mathcal{M}_{g} /\left[\mathcal{M}_{g}, \mathcal{M}_{g}\right]$ is isomorphic to $\mathbb{Z}_{2} \oplus \mathbb{Z}_{2}$ for $g=2$ and to $\mathbb{Z}_{2}$ for $g>2$.

Proof. Elements conjugate in $\mathcal{M}_{g}$ become equal in $A$. By (P7) all elements $a_{i}$ become equal in $A$ to an element which we denote by $a$. We have $r_{1,2} * a_{2}=d_{1,2}$ by (P10), and by Definition 3 all $d_{i, j}$ with $i \neq-j$ become equal to $a$ in $A$. By (P5) all elements $t_{i}$ become equal in $A$ to an element which we denote by $t$. Also all elements $r_{i, j}$ are equal to $t$ in $A$ by (P11). All elements $s_{i}$ become equal in $A$ to an element which we denote by $s$. So $A$ is generated by $t, s$ and $a$. Now $t^{2}=s^{2}$ by (P9), and $t^{2}=a^{-2}$ 
by (P6). Consider the relation in (P12) defining $r_{1,3}$. Reducing modulo the commutator we get $t=s^{3} t^{-2} a$ in $A$. Since $t^{2}=s^{2}$ we get $t=s a$. Squaring both sides we get $t^{2}=s^{2} a^{2}$, hence $a^{2}=t^{2}=s^{2}=1$. So $A$ is generated by $a$ and $s$ and $a^{2}=s^{2}=1$.

We now consider separately the cases $g=2$ and $g>2$. If $g=2$ then all edges of $X$ are equivalent to $\mathbf{e}_{1,2}$. The relations (P12) become empty. We have very few standard curves and very few corresponding elements $d_{I}: d_{I_{0}}$, $d_{I}$, where $I=I_{0}-k$ for $k \in I_{0}$, and $d_{i, j}$ with $i<j$. In $A$ the elements $d_{-1,1}$ and $d_{-2,2}$ become trivial by the definition and by the previous relations. We have seen that $d_{i, j}$ becomes equal to $a$ for $i \neq-j$ so $d_{I_{0}}$ is equal to 1 in $A$ and $d_{I}$ is equal to $a$ in $A$ for $I=I_{0}-k$. All relations (P1)-(P11) become trivial modulo the commutator and the relations $a^{2}=s^{2}=1$. Thus $A=\mathbb{Z}_{2} \oplus \mathbb{Z}_{2}$.

Consider now the case $g>2$. From the definition of $c_{1,3}$ and from the previous relations we get $c_{1,3}=1$ in $A$. By (P10)(a) we get $a=1$, so $A$ is generated by $s$ and $s^{2}=1$. Now all relations (P1)-(P12) become trivial so $A=\mathbb{Z}_{2}$.

2.1. The stabilizer of a vertex. The main task of this subsection is to establish a presentation of the stabilizer $H$ of the vertex $v_{0}$ of $X$.

Proposition 21. The group $H$ admits a presentation with generators $a_{i}, i=1, \ldots, g, d_{1,2}, s$ and $t_{i}, i=1, \ldots, g-1$, and with relations $(\mathrm{P} 1)-(\mathrm{P} 8)$ of Theorem 18.

The group $H$ can be defined by two exact sequences:

$$
\begin{gathered}
1 \rightarrow \mathbb{Z}_{2}^{g} \rightarrow \pm \Sigma_{g} \rightarrow \Sigma_{g} \rightarrow 1, \\
1 \rightarrow H_{0} \rightarrow H \rightarrow \pm \Sigma_{g} \rightarrow 1 .
\end{gathered}
$$

Before defining the objects and the homomorphisms in these sequences we explain how from an exact sequence $1 \rightarrow A \rightarrow B \rightarrow C \rightarrow 1$ and from finite presentations of the groups $A$ and $C$ we can construct a finite presentation of $B$.

- We write generators $a_{i}$ and relations $Q_{j}$ in $A$.

- We write generators $c_{i}$ and relations $W_{j}$ in $C$.

- We lift generators $c_{i}$ to elements $b_{i}$ in $B$.

- We substitute $b_{i}$ for $c_{i}$ in $W_{j}$ getting a word $R_{j}$ in the letters $b_{i}$, which represents an element $d_{j}$ of $A$. We write $d_{j}$ as a product of $a_{i}$ 's.

- Finally, for every $a_{i}$ and $b_{j}$ the conjugate $b_{j} * a_{i}$ represents an element $a_{i, j}$ of $A$, which we write as a product of the generators $a_{i}$.

- $B$ has a presentation with generators $a_{i}$ and $b_{j}$ and with relations $Q_{i}=1, R_{j}=d_{j}$, and $b_{j} a_{i} b_{j}^{-1}=a_{i, j}$. 
We now describe the sequence 1 and the group $\pm \Sigma_{g}$. This is the group of permutations of the set $I_{0}=\{-g, 1-g, \ldots,-1,1,2, \ldots, g\}$ such that $\sigma(-i)=-\sigma(i)$. The homomorphism $\pm \Sigma_{g} \rightarrow \Sigma_{g}$ forgets the signs. A generator of the kernel changes the sign of one letter. The sequence splits, $\Sigma_{g}$ can be considered as the subgroup of the permutations which take positive numbers to positive numbers. Let $\tau_{i}=(i, i+1)$ be a transposition in $\Sigma_{g}$ for $i=1, \ldots, g-1$. Then

(S1) $\left[\tau_{i}, \tau_{j}\right]=1$ for $|i-j|>1$,

(S2) $\tau_{i} * \tau_{i+1}=\tau_{i+1}^{-1} * \tau_{i}$ for $i=1, \ldots, g-2$,

(S3) $\tau_{i}^{2}=1$ for $i=1, \ldots, g-1$.

This defines a presentation of $\Sigma_{g}$. Further, let $\sigma_{i}$ for $i=1, \ldots, g$ denote the change of sign of the $i$ th letter in a signed permutation. Then $\sigma_{i}^{2}=1$ and $\left[\sigma_{i}, \sigma_{j}\right]=1$ for all $i, j$. Finally, conjugation gives $\tau_{i} * \sigma_{i}=\sigma_{i+1}, \tau_{i} * \sigma_{i+1}=\sigma_{i}$ and $\left[\sigma_{j}, \tau_{i}\right]=1$ for $j \neq i$ and $j \neq i+1$. In fact, it suffices to use one generator $\sigma=\sigma_{1}$ and the relations $\sigma_{i}=\left(\tau_{i-1} \tau_{i-2} \ldots \tau_{1}\right) * \sigma$. We get the relations

(S4) $\sigma^{2}=1$

(S5) $\left[\left(\tau_{i} \tau_{i-1} \ldots \tau_{1}\right) * \sigma, \tau_{j}\right]=1$ for $j \neq i$ and $j \neq i+1$,

(S6) $\left[\left(\tau_{i} \tau_{i-1} \ldots \tau_{1}\right) * \sigma,\left(\tau_{j} \tau_{j-1} \ldots \tau_{1}\right) * \sigma\right]=1$ for all $i, j$.

The group $\pm \Sigma_{g}$ has a presentation with generators $\sigma, \tau_{1}, \ldots, \tau_{g-1}$ and with defining relations $(\mathrm{S} 1)-(\mathrm{S} 6)$.

We now describe the sequence (2). A homeomorphism in $H$ may permute the curves $\alpha_{i}$ and may change their orientations. We fix an orientation of each curve $\alpha_{i}$ and define a homomorphism $\phi_{1}: H \rightarrow \pm \Sigma_{g}$ as follows: a homeomorphism $h$ is mapped to a permutation $i \mapsto \pm j$ if $h\left(\alpha_{i}\right)=\alpha_{j}$ and the sign is "+" if the orientations of $h\left(\alpha_{i}\right)$ and of $\alpha_{j}$ agree, and "-" otherwise. If $h$ preserves the isotopy class of $\alpha_{i}$ and preserves its orientation then it is isotopic to a homeomorphism fixed on $\alpha_{i}$. The kernel of $\phi_{1}$ is the subgroup $H_{0}$ of the elements of $H$ represented by the homeomorphisms which keep the curves $\alpha_{1}, \ldots, \alpha_{g}$ pointwise fixed. Such a homeomorphism induces a homeomorphism of the surface $S_{0}$ obtained by cutting $S$ open along the curves $\alpha_{i}$.

We shall study the mapping class group of $S_{0}$ and from it we shall deduce a presentation of $H_{0}$. We want to make the presentation symmetric with respect to all holes so it is relatively complicated but it will simplify the subsequent treatment of the mapping class group $\mathcal{M}$. We use the notation from Definition 3.

LEMma 22. The mapping class group of $S_{0}$ has a presentation with generators $d_{i}, i \in I_{0}$, and $d_{i, j}, i<j \in I_{0}$, and with relations

(Q1) $\left[d_{i}, d_{j}\right]=1$ and $\left[d_{i}, d_{j, k}\right]=1$ for all $i, j, k \in I_{0}$,

(Q2) pure braid relations 
(a) $d_{r, s}^{-1} * d_{i, j}=d_{i, j}$ if $r<s<i<j \in I_{0}$ or $i<r<s<j \in I_{0}$,

(b) $d_{r, i}^{-1} * d_{i, j}=d_{r, j} * d_{i, j}$ if $r<i<j \in I_{0}$,

(c) $d_{i, s}^{-1} * d_{i, j}=\left(d_{i, j} d_{s, j}\right) * d_{i, j}$ if $i<s<j \in I_{0}$,

(d) $d_{r, s}^{-1} * d_{i, j}=\left[d_{r, j}, d_{s, j}\right] * d_{i, j}$ if $r<i<s<j \in I_{0}$,

(Q3) $d_{I_{0}}=1$,

(Q4) $d_{k}=d_{I_{k}}$ for every $k \in I_{0}$ where $I_{k}=I_{0}-\{k\}$.

Proof. We start with a big disk $D$ in $S_{0}$ which contains all holes $\partial_{i}$, $i \in I_{0}$. When we glue a disk with a distinguished centre to each curve $\partial_{i}$ we get a disk with $2 g$ distinguished points. Its mapping class group is isomorphic to the pure braid group $P_{2 g}$ with generators $d_{i, j}$ and with relations (Q2). In the passage from the mapping class group of $D$ to the mapping class group of the punctured disk we kill exactly the twists $d_{i}$, which commute with everything. One can check that the removal of the disks does not affect the relations (Q2) so the mapping class group of $D$ has a presentation with relations (Q1) and (Q2). Now we glue a disk with a distinguished centre $P$ to the boundary $\partial$ of $D$. This kills the twist $d$ around the boundary $\partial$ and we get (Q3), by Lemma 17. Finally, we forget the distinguished point $p$ and get the sphere $S_{0}$. By [2], Theorem 4.3, the kernel of the homomorphism induced by this forgetful map is generated by spin maps with respect to some set of generators of $\pi_{1}\left(S_{0}, P\right)$.

We can choose a base point $P$ on the $x$-axis to the right of all the holes and choose generators of $\pi_{1}\left(S_{0}, P\right)$ to be simple loops $\lambda_{k}$ such that $\lambda_{k}$ moves parallel to the $x$-axis, turns around the hole $\partial_{k}$ and comes back to $P$ parallel to the $x$-axis (see Figure 10). A spin map with respect to $\lambda_{k}$ is isotopic to a quotient of twists with respect to the boundaries of a regular neighbourhood of $\lambda_{k}$. We can homotope these boundaries into $D$, without passing over $P$. Then one is isotopic to $\partial_{k}$ and the other is isotopic to the standard curve $\delta_{I}$ with $I=I_{0}-\{k\}$. By Lemma 17 we get (Q4).

We now go back to the presentation of $H_{0}$. When we glue back the corresponding pairs of boundary components of $S_{0}$ we get the surface $S$. This glueing map induces a homomorphism from the mapping class group of $S_{0}$ into $H_{0}$. In fact, it is onto $H_{0}$-a homeomorphism fixed on $\alpha_{1}, \ldots, \alpha_{g}$ induces a homeomorphism of $S_{0}$. One can prove that the kernel is generated by the products $d_{i} d_{-i}^{-1}$ so both twists are identified with $a_{i}$ in $H_{0}$. Considering the presentation of the mapping class group of $S_{0}$ established in Lemma 22 we see that $H_{0}$ has a presentation with generators $a_{1}, \ldots, a_{g}$ and $d_{i, j}$ for $i<j \in I_{0}$ and with relations (P1)-(P4). In these relations $d_{i, j}$ is represented by a Dehn twist along $\delta_{i, j}$.

We now lift the generators of $\pm \Sigma_{g}$. Recall that $t_{i}\left(\alpha_{i}\right)=\alpha_{i+1}$, and $t_{i}\left(\alpha_{i+1}\right)=\alpha_{i}$, and $t_{i}\left(\alpha_{k}\right)=\alpha_{k}$ for $k \neq i, i+1$. $s\left(\alpha_{1}\right)$ is equal to $\alpha_{1}$ with the 
opposite orientation and $s$ is fixed on the other $\alpha_{i}$ 's. Thus we can lift $\tau_{i}$ to $t_{i}$ and $\sigma$ to $s$.

When we lift the relations in $\pm \Sigma_{g}$ and establish the conjugates of the generators of $H_{0}$ by the lifted generators of $\pm \Sigma_{g}$ we get a presentation of $H$. In order to prove Proposition 21 we consider an abstract group defined by the generators and relations in Proposition 21. We map this group onto $H$ where $a_{i}, d_{1,2}, s$ and $t_{i}$ are mapped onto corresponding elements in $H$. By the previous discussion the products $d_{i, j}$ from Definition 3 are mapped onto corresponding twists. Therefore relations $(\mathrm{P} 1)-(\mathrm{P} 4)$ are mapped onto true relations in $H$. Now we check that (P5)-(P8) are also mapped onto true relations in $H$. We use Lemma 15 and relations (i)-(iv) of Lemma 16.

Relation (P5) follows from the definition of $t_{i}$ and from (i) and (iv).

We already know that $d_{-1,1}=s^{2} a_{1}^{4}$. By (i) and (ii), $t_{i}^{2} a_{i+1}^{2} a_{i}^{2}=\left(a_{i} e_{i} a_{i+1}\right)^{4}$ for $i<g$. The boundary of a regular neighbourhood of $\alpha_{i} \cup \varepsilon_{i} \cup \alpha_{i+1}$ is equal to $\delta_{i, i+1} \cup \delta_{-i-1,-i}$. Thus (P6) follows from (iii).

The relations in (P7) follow easily from Lemma 15 and (i).

Since $t_{1}\left(\alpha_{1}\right)=\alpha_{2}$ and $s\left(\alpha_{2}\right)=\alpha_{2}$ we have $\left[t_{1} s t_{1}, a_{1}\right]=1$ by Lemma 15 . Also $t_{1} s t_{1}=e_{1} a_{1} b_{1} a_{2} e_{1} a_{1}^{2} e_{1} a_{2} b_{1} a_{1} b_{1}$ by (i) and (ii), and the last expression commutes with $b_{1}$ by (i) and (ii), hence it commutes with $s . d_{-2,2}$ is supported inside $\delta_{-2,2}$ and $s$ is supported inside $\delta_{-1,1}$, so they commute. We have further $\left[s, d_{2,3}\right]=1$ and $\left[t_{i}, d_{1,2}\right]=1$ for $i \neq 2$ by (i). Thus (P8) is also satisfied in $H$.

It remains to lift the relations of $\pm \Sigma_{g}$ to $H$, compute the conjugation relations of the generators of $H_{0}$ by the lifted generators of $\pm \Sigma_{g}$, and prove that all these relations follow from (P1)-(P8).

Relations (S1) and (S2) lift to (P5).

Relations (S3) and (S4) lift to (P6).

Relation (S5) lifts to $\left[\left(t_{i} t_{i-1} \ldots t_{1}\right) * s, t_{j}\right]=1$ for $j \neq i$ and $j \neq i+1$ and this follows from (P5) and (P7).

We shall deal with (S6) a little later. We now pass to the conjugation by $s$ and $t_{k}$. Since $s^{2} \in H_{0}$ and $t_{k}^{2} \in H_{0}$ by (P6), it suffices to know the result of the conjugation of each generator of $H_{0}$ by either $s$ or $s^{-1}$, and by either $t_{k}$ or $t_{k}^{-1}$ (the other follows). We have $s * a_{i}=a_{i}$ by (P7). The result of the conjugation $t_{j} * a_{i}$ is determined by $(\mathrm{P} 7)$.

We now prove that the result of the conjugation of $d_{i, j}$, by either $t_{k}$ or $t_{k}^{-1}$, and by either $s$ or $s^{-1}$, is determined by (P5), (P7), (P8) and the definitions of $d_{i, j}$. Consider first the conjugation by $s$.

If $i>1$ then $d_{i, j}=\left(t_{i-1} \ldots t_{2} t_{j-1} \ldots t_{3}\right) * d_{2,3}$ by (P5), so $s * d_{i, j}=d_{i, j}$ by (P7) and (P8).

If $i=1$ then $s^{-1} * d_{1, j}=d_{-1, j}$ by (P7) and the definitions. 
If $i<-1$ and $j+i>0$ then $d_{i, j}=\left(t_{-i-1}^{-1} \ldots t_{2}^{-1} t_{j-1} \ldots t_{3} t_{1}^{-1} s^{-1} t_{2}\right) * d_{1,2}$ by (P5) and (P7). Now

$$
\begin{aligned}
\left(s^{-1} t_{1}^{-1} s^{-1} t_{2}\right) * d_{1,2} & \left.=s^{-1} t_{1}^{-1} s^{-1} t_{1}^{-1}\right) * d_{2,3} \\
& =\left(t_{1}^{-1} s^{-1} t_{1}^{-1}\right) * d_{2,3} \quad(\text { by }(\mathrm{P} 7) \text { and }(\mathrm{P} 8)) \\
& =\left(t_{1}^{-1} s^{-1} t_{2}\right) * d_{1,2} .
\end{aligned}
$$

Thus $s^{-1} * d_{i, j}=d_{i, j}$.

The case of $i<-1$ and $j>1$ and $i+j<0$ is similar.

If $j=-1$ then $d_{i, j}=\left(t_{-i-1}^{-1} \ldots t_{1}^{-1} s^{-1} t_{1}^{-1} s^{-1}\right) * d_{1,2}$. Now

$$
\left(s t_{1}^{-1} s^{-1} t_{1}^{-1} s^{-1}\right) * d_{1,2}=\left(t_{1}^{-1} s^{-1}\right) * d_{1,2} \quad(\text { by }(\mathrm{P} 7))
$$

hence $s * d_{i,-1}=d_{i, 1}$.

Now

If $j<-1$ then $d_{i, j}=\left(t_{-j-1}^{-1} \ldots t_{2}^{-1} t_{-i-1}^{-1} \ldots t_{3}^{-1} t_{1}^{-1} t_{2}^{-1} s^{-1} t_{1}^{-1} s^{-1}\right) * d_{1,2}$.

$$
\begin{aligned}
s^{-1} *\left(\left(t_{1}^{-1} t_{2}^{-1} s^{-1}\right.\right. & \left.\left.t_{1}^{-1} s^{-1}\right) * d_{1,2}\right) \\
& =\left(s^{-1} t_{1}^{-1} s^{-1} t_{1}^{-1} t_{1} t_{2}^{-1} t_{1}^{-1} s^{-1}\right) * d_{1,2} \\
& =\left(t_{1}^{-1} s^{-1} t_{1}^{-1} s^{-1} t_{2}^{-1} t_{1}^{-1} t_{2} s^{-1}\right) * d_{1,2} \\
& \left.=\left(t_{1}^{-1} s^{-1} t_{1}^{-1} t_{2}^{-1}\right) *\left(s^{-1} t_{1}^{-1} s^{-1} t_{2}\right) * d_{1,2}\right) \\
& =\left(t_{1}^{-1} s^{-1} t_{1}^{-1} t_{2}^{-1} t_{1}^{-1} s^{-1} t_{2}\right) * d_{1,2} \quad \text { (as before) } \\
& =\left(t_{1}^{-1} s^{-1} t_{2}^{-1} t_{1}^{-1} s^{-1}\right) * d_{1,2} \quad \text { (by (P7) and (P5)). }
\end{aligned}
$$

Hence $s^{-1} * d_{i, j}=d_{i, j}$.

If $i=-j$ then $s * d_{k, k+1}=d_{k, k+1}$ for $k>1$, as above, hence $s * d_{i, j}=$ $\left(t_{j-1}^{-1} d_{j-1, j} \ldots t_{2}^{-1} d_{2,3} s\right) * d_{-2,2}=d_{i, j}$ by $(\mathrm{P} 7)$.

We now check conjugation by $t_{k}$. If $i \neq-j$ then it follows easily from (P5), (P7) and (P8) that $d_{i, j}$ either remains invariant under the conjugation, or is transformed into a "neighbouring" $d_{p, q}$. In particular, $t_{k}$ commutes with $d_{i, i+1}$ if $k<i-1$ or $k=i$ or $k>i+1$. Now, by (P5) and the definitions, $t_{k} * d_{-j, j}=d_{-j, j}$ if $k \neq j-1, j, t_{j}^{-1} * d_{-j, j}=d_{j, j+1}^{-1} * d_{-j, j}$ and $t_{j-1} * d_{-j, j}=d_{j-1, j} * d_{-j, j}$.

Finally, we lift $(\mathrm{S} 6)$. We start with the case $\left[\tau_{1} * \xi_{1}, \xi_{1}\right]$. It lifts to

$$
\begin{aligned}
t_{1} s t_{1}^{-1} s t_{1} s^{-1} t_{1}^{-1} s^{-1} & =t_{1} s t_{1}^{-1} s s^{-1} t_{1}^{-1} s^{-1} t_{1} \quad \text { (by (P7)) } \\
& =t_{1} s t_{1}^{-2} s^{-1} t_{1}^{-1} t_{1}^{2} .
\end{aligned}
$$

We have $t_{1}^{2} \in H_{0}$ by (P6), and the conjugation of an element of $H_{0}$ by $s$ and $t_{i}$ is already determined by $(\mathrm{P} 5)-(\mathrm{P} 8)$. So we know how to lift $\left[\tau_{1} * \xi_{1}, \xi_{1}\right]=1$. In the general case we have a commutator $\left[\left(t_{i} t_{i-1} \ldots t_{1}\right) * s,\left(t_{j} t_{j-1} \ldots t_{1}\right) * s\right]$. If $i>j$ then, by (P5) and (P7), this commutator is equal to the conjugate 
of $\left[t_{1} * s, s\right]$ by $t_{j} t_{j-1} \ldots t_{1} t_{i} t_{i-1} \ldots t_{2}$. This is a conjugation of an element of $H_{0}$ by $t_{k}$ 's so the result is determined by (P5)-(P8).

This concludes the proof of Proposition 21.

2.2. Proof of the main theorem. We now return to the action of $\mathcal{M}$ on $X$. A homeomorphism $h$ of $B$ takes meridian curves to meridian curves and cut-systems to cut-systems. If we let $h\left(\left\langle\gamma_{1}, \ldots, \gamma_{g}\right\rangle\right)=\left\langle h\left(\gamma_{1}\right), \ldots, h\left(\gamma_{g}\right)\right\rangle$ we get an action of $\mathcal{M}$ on the vertices of $X$ which extends to a simplicial action on $X$. Conversely, if $h$ is a homeomorphism of $S$ which takes a cut-system $v=\left\langle\gamma_{1}, \ldots, \gamma_{g}\right\rangle$ onto a cut-system $w$ then $h$ belongs to $\mathcal{M}$ ( $h$ can be extended to the interior of $B$ ).

We now sketch the rest of the proof of Theorem 18. We prove that $\mathcal{M}$ acts transitively on the vertices of $X$ and that there are only a finite number of edge-orbits of the action. We choose representatives $\mathbf{e}_{i, j}$ of the edge-orbits and prove that the chosen element $r_{i, j}$ of $\mathcal{M}$ switches the vertices of $\mathbf{e}_{i, j}$. We prove that $\mathcal{M}$ is generated by $r_{i, j}$ 's together with the generators of $H$.

Now to every word in the generators we assign an edge-path in $X$. To a word representing the identity in $\mathcal{M}$ corresponds a closed edge-path. We establish relations between words which induce the same edge-path or edgepaths which differ by cancelling trivial "tails" and we prove that the relations follow from (P9) and (P10). We show that there are only a finite number of face-orbits of the action of $\mathcal{M}$ on $X$. We choose representatives of face-orbits and for each of them we choose a word which represents the identity in $\mathcal{M}$ and to which corresponds the boundary edge-path of the face. These words correspond to (P11) and (P12). Now if a word $w$ in the generators represents the identity in $\mathcal{M}$ then it induces a closed edge-path. By Theorem 1 this path is a sum of triangular paths (with tails). It follows easily that $w$ is a product of the relators $(\mathrm{P} 1)-(\mathrm{P} 12)$.

We now prove that $\mathcal{M}$ acts transitively on the vertices of $X$. If $v$ and $w$ are cut-systems and we cut $S$ open along the curves of $v$ (respectively $w)$ we get a sphere with $2 g$ holes. We can construct a homeomorphism of one sphere onto the other which respects the identification of the boundary components corresponding to the same curve of $v$ (respectively $w$ ). It induces a homeomorphism of $S$ which takes $v$ onto $w$ and thus belongs to $\mathcal{M}$.

We want to classify the edges of $X$ modulo the action of $\mathcal{M}$. Let $\mathbf{e}=$ $(v, w)$ be an edge of $X$ connecting the vertices $v$ and $w$. When we cut $S$ open along the curves of $v$ we get a sphere $S_{1}$ with $2 g$ holes. The vertex $w$ has one new curve $\gamma$ disjoint from the curves of $v$. When we cut $S_{1}$ along $\gamma$ we get two components $D_{1}$ and $D_{2}$ homeomorphic to spheres with holes. We may assume that $D_{1}$ has at most $g+1$ holes. Some of its holes come in pairs corresponding to the same curve of $v$ and some are single holes, with the corresponding holes in $D_{2}$. Let now $\mathbf{e}^{\prime}=\left(v^{\prime}, w^{\prime}\right)$ be another edge 
and let $D_{1}^{\prime}$ and $D_{2}^{\prime}$ be the corresponding spheres with holes. There exists a homeomorphism $h$ of $S$ which takes $v$ onto $v^{\prime}$ and $w$ onto $w^{\prime}$ (and thus induces a homeomorphism of $D_{1}$ onto $D_{1}^{\prime}$ ) if and only if the numbers of pairs of holes and of single holes in $D_{1}$ are the same as in $D_{1}^{\prime}$.

Consider the vertex $v_{0}=\left\langle\alpha_{1}, \ldots, \alpha_{g}\right\rangle$ which was fixed in the previous section. For any pair of natural numbers $p, q$ such that $2 p+q \leq g$ there exists a unique curve $\gamma_{i, j}$ (see Definition 2) such that $\gamma_{i, j}$ contains inside exactly $p$ pairs of holes of $S_{0}$ and $q$ single holes of $S_{0}$ and $i<j \in I_{0}$ and $i=1$ or $(i<0$, $i+j>0, j-i \leq g)$. Figure 12 shows a curve $\gamma_{i, j-1}$ for $i<0$. If we replace a curve $\alpha_{j}$ of the cut-system $v_{0}=\left\langle\alpha_{1}, \ldots, \alpha_{g}\right\rangle$ by the curve $\gamma_{i, j}$ we get another cut-system $v_{i, j}$ which is connected to $v_{0}$ by an edge $\mathbf{e}_{i, j}=\left(v_{0}, v_{i, j}\right)$ in $X$. By the above argument every edge $\mathbf{e}$ of $X$ can be mapped by an element of $\mathcal{M}$ onto the unique edge $\mathbf{e}_{i, j}$. We say that e has type $(i, j)$.

For each edge type $(i, j)$ with $i, j \in I_{0}, i \leq 1, i+j>0, j-i \leq g$, we have chosen an element $r_{i, j}=b_{j} a_{j} c_{i, j} b_{j}$ of $\mathcal{M}$. By Lemma 16(ii), $r_{i, j}\left(\alpha_{j}\right)=\gamma_{i, j}$, $r_{i, j}\left(\gamma_{i, j}\right)=\alpha_{j}$ and clearly $r_{i, j}\left(\alpha_{k}\right)=\alpha_{k}$ for $k \neq j$. Thus $r_{i, j}\left(v_{0}\right)=v_{i, j}$, $r_{i, j}\left(v_{i, j}\right)=v_{0}$ and $r_{i, j}^{2} \in H$.

We now describe precisely a construction from [11] and [7] which will let us determine a presentation of $\mathcal{M}$.

To every edge-path $\mathbf{p}=\left(v_{0}, v_{1}, \ldots, v_{k}\right)$ which begins at $v_{0}$ we assign a product of elements of $\mathcal{M}$ of the form $g=h_{1} r_{1} h_{2} r_{2} \ldots h_{k} r_{k} h_{k+1}$ such that for $m=1, \ldots, k$ we have $h_{1} r_{1} \ldots h_{m} r_{m}\left(v_{0}\right)=v_{m}$, where $h_{m} \in H$ and $r_{m}=r_{i, j}$ if the edge $\left(v_{m-1}, v_{m}\right)$ has type $(i, j)$. We call such a product an $h$-product corresponding to $\mathbf{p}$. We construct it as follows. If the edge $\left(v_{0}, v_{1}\right)$ has type $(i, j)$ then there exists $h_{1} \in \mathcal{M}$ such that $h_{1}\left(v_{0}\right)=v_{0}$ and $h_{1}\left(v_{i, j}\right)=v_{1}$. Then for $r_{1}=r_{i, j}$ we have $h_{1} r_{1}\left(v_{0}\right)=v_{1}$. Next we transport the second edge to $v_{0}$. We have $\left(h_{1} r_{1}\right)^{-1}\left(v_{1}\right)=v_{0}$ and $\left(h_{1} r_{1}\right)^{-1}\left(v_{2}\right)=v_{1}^{\prime}$. If the second edge has type $(p, q)$ and we let $r_{2}=r_{p, q}$ then there exists $h_{2} \in H$ such that $h_{2} r_{2}\left(v_{0}\right)=v_{1}^{\prime}$ and $h_{1} r_{1} h_{2} r_{2}\left(v_{0}\right)=v_{2}$, and so on. Observe that the elements $h_{i}$ in the $h$-product corresponding to an edge-path $\mathbf{p}$ are not uniquely determined. The construction implies

Lemma 23. The generators of $H$ together with the elements $r_{i, j}$ generate the group $\mathcal{M}$.

Proof. Let $g \in \mathcal{M}$. Then $g\left(v_{0}\right)$ is a vertex of $X$ and it can be connected to $v_{0}$ by an edge-path $\mathbf{p}=\left(v_{0}, v_{1}, \ldots, v_{k}=g\left(v_{0}\right)\right)$. Let $g_{1}=$ $h_{1} r_{1} h_{2} r_{2} \ldots h_{k} r_{k}$ be an $h$-product corresponding to $\mathbf{p}$. Then $g_{1}\left(v_{0}\right)=v_{k}=$ $g\left(v_{0}\right)$, therefore $g^{-1} g_{1}$ leaves $v_{0}$ fixed and belongs to the stabilizer $H$ of $v_{0}$. It follows that $g=h_{1} r_{1} h_{2} r_{2} \ldots h_{k} r_{k} h_{k+1}$.

By the inverse process we define an edge-path induced by the $h$-product $g=h_{1} r_{1} h_{2} r_{2} \ldots h_{k} r_{k} h_{k+1}$. The edge-path starts at $v_{0}$, then $v_{1}=h_{1} r_{1}\left(v_{0}\right)$, $v_{2}=h_{1} r_{1} h_{2} r_{2}\left(v_{0}\right)$ and so on. The last vertex of the path is $v_{k}=g\left(v_{0}\right)$. 
REMARK 2. An $h$-product $g$ represents an element in $H$ if and only if $v_{k}=v_{0}$. This happens if and only if the corresponding edge-path is closed. We can multiply such a $g$ by a suitable element of $H$ on the right and get an $h$-product which represents the identity in $\mathcal{M}$ and induces the same edge-path as $g$.

We now prove that relations (P9) are satisfied in $\mathcal{M}$.

Proof. By Lemma 16(i), (ii),

$$
\begin{aligned}
r_{i, j} & =b_{j} a_{j} c_{i, j} b_{j}^{2} a_{j} c_{i, j} b_{j}=s_{j} b_{j}^{-1} a_{j}^{-1} c_{i, j} b_{j}^{2} a_{j} c_{i, j} b_{j} \\
& =s_{j} b_{j}^{-1} c_{i, j} a_{j}^{-1} b_{j}^{2} a_{j} c_{i, j} b_{j}=s_{j} b_{j}^{-1} c_{i, j} b_{j} a_{j}^{2} b_{j}^{-1} c_{i, j} b_{j} \\
& =s_{j} c_{i, j} b_{j} c_{i, j}^{-1} a_{j}^{2} c_{i, j} b_{j} c_{i, j}^{-1}=s_{j} c_{i, j} s_{j} c_{i, j}^{-1} .
\end{aligned}
$$

We next prove that relations $(\mathrm{P} 10)$ are satisfied in $\mathcal{M}$. For a fixed edge type $(i, j)$ they are related to the stabilizer $H_{i, j}$ of the edge $\mathbf{e}_{i, j}$ in $H$. Every element $h \in H_{i, j}$ takes $\alpha_{j}$ onto itself and takes $\gamma_{i, j}$ onto itself, and permutes the other $\alpha_{i}$ 's. Since $r_{i, j}$ switches $\alpha_{j}$ and $\gamma_{i, j}$ and is fixed on the other $\alpha_{k}$ 's we have $r_{i, j} h r_{i, j}^{-1} \in H_{i, j}$. To make this statement into a finite set of relations we must find a finite set of generators for $H_{i, j}$ and find the corresponding conjugate of each generator.

Lemma 24. The group $H_{i, j}$ which stabilizes the edge $\mathbf{e}_{i, j}$ is generated by the following elements:

- $a_{1}, \ldots, a_{g}$,

- $t_{k}$ where $k<|i|$, or $k>j$, or $|i|<k<j-1$, or $k=i=1<j-1$,

- $s_{k}$ where $k<|i|$, or $k>j$, or $k=-i>0$,

- $d_{k, m}$ where $k, m \in\{p \mid i \leq p \leq j\}$ or $k, m \in\{p \mid p<i$ or $j<p\}$,

- $z_{j}$ if $i=1$ and $j=g$, or $i<0$ and $j-i=g$.

P r o of. A homeomorphism $h$ in $H_{i, j}$ takes $\gamma_{i, j}$ onto itself. If $\gamma_{i, j}$ contains exactly $g$ holes then $h$ may switch the two components of $S_{0}-\gamma_{i, j}$. This can be done by the homeomorphism $z_{j}$, which preserves $\gamma_{i, j}$ and $\alpha_{j}$. Modulo $z_{j}$ we may assume that $h$ leaves each component of $S_{0}-\gamma_{i, j}$ invariant. It must leave $\partial_{j}$ fixed, and may switch $\partial_{k}$ and $\partial_{-k}$ if they are in the same component of $S_{0}-\gamma_{i, j}$. This corresponds to the reversal of the orientation of $\alpha_{k}$ and can be done by $s_{k}$. Also, $h$ may permute single holes in a component of $S_{0}-\gamma_{i, j}$ (and permute in the same way the corresponding holes in the other component). It may also permute pairs of holes $\partial_{k}, \partial_{-k}$ lying in the same component of $S_{0}-\gamma_{i, j}$. All these permutations are induced by the suitable $t_{k}$ 's (see Definition 4). Modulo these homeomorphisms we may assume that $h$ is fixed on all curves $\partial_{k}$. The restriction of $h$ to each component of $S_{0}-\gamma_{i, j}$ belongs to the mapping class group of the component. As in Lemma 22 each mapping class group is generated by the twists $d_{i}$ for $\partial_{i}$ in the component 
and the twists $d_{k, m}$ for $\delta_{k, m}$ in the component. (The curves $\delta_{k, m}$ with $k<i$ and $m>j$ can be pushed over the point at infinity and drawn around the right hand side of Figure 10. Then they look more like the curves in Figure 8 containing two holes in the component outside $\gamma_{i, j}$.)

We now have to compute the result of the conjugation of the generators of $H_{i, j}$ by $r_{i, j}$.

Lemma 25. Relations (P10) are satisfied in $\mathcal{M}$ and they determine the result (in $H$ ) of the conjugation of the generators of $H_{i, j}$ by $r_{i, j}$.

Pro of. Relations (a)-(d) of (P10) follow immediately from the definitions and from Lemma 16(i), (ii).

$z_{j}$ takes each of the curves $\alpha_{j}, \beta_{j}$, and $\gamma_{i, j}$ onto itself so it commutes with $r_{i, j}$ by Lemma 15.

The last four relations follow by Lemma 15. One has to draw several pictures to prove it. Since $r_{i, j}^{2} \in H$ (by (P9)) it suffices to know the result of the conjugation of each generator of $H_{i, j}$ by either $r_{i, j}$ or $r_{i, j}^{-1}$. Not all generators $d_{k, m}$ from Lemma 24 appear in the last four relations of (P10) but the other generators are obtained from these by conjugation by suitable products of $s$ and $t_{k}$ 's which are already in $H_{i, j}$.

From (P9) and (P10) we get information about $h$-products.

Claim 1. If two h-products represent the same element in $\mathcal{M}$ and induce the same edge-path then they are equivalent modulo (P1)-(P10).

P roof. If two $h$-products $g_{1}=h_{1} r_{1} \ldots r_{k} h_{k+1}$ and $g_{2}=f_{1} r_{1}^{\prime} \ldots r_{k}^{\prime} f_{k+1}$ induce the same edge-path $\mathbf{p}=\left(v_{0}, v_{1}, \ldots, v_{k}\right)$ then $h_{1} r_{1}\left(v_{0}\right)=f_{1} r_{1}^{\prime}\left(v_{0}\right)$. Therefore $h_{1}^{-1} f_{1} r_{1}^{\prime}\left(v_{0}\right)=r_{1}\left(v_{0}\right)$. Since $h_{1}^{-1} f_{1}$ preserves the edge type of the edge $\left(v_{0}, r_{1}^{\prime}\left(v_{0}\right)\right)$, say $(i, j)$, we have $r_{1}=r_{1}^{\prime}=r_{i, j}$ and $h_{1}^{-1} f_{1} \in H_{i, j}$. By (P10) we have $f_{1} r_{1}^{\prime} f_{2}=h_{1} h_{1}^{-1} f_{1} r_{1} f_{2}=h_{1} r_{1} h_{1}^{\prime} f_{2}=h_{1} r_{1} f_{2}^{\prime}$. Therefore $g_{2}$ is equivalent to a new $h$-product $h_{1} r_{1} f_{2}^{\prime} r_{2}^{\prime} f_{3} r_{3}^{\prime} \ldots r_{k}^{\prime} f_{k+1}$ which induces the same edge-path $\mathbf{p}$. If we apply $r_{1}^{-1} h_{1}^{-1}$ to the vertices $\left(v_{1}, \ldots, v_{k}\right)$ of $\mathbf{p}$ we get a shorter edge-path which starts at $v_{0}$ and is induced by two shorter $h$-products $h_{2} r_{2} \ldots r_{k} h_{k+1}$ and $f_{2}^{\prime} r_{2}^{\prime} \ldots r_{k}^{\prime} f_{k+1}$. Claim 1 follows by induction on $k$.

Two different edge-paths may be homotopic in the 1-skeleton $X^{1}$. This means that there is a backtracking $v_{i}, v_{i+1}, v_{i}$ along the edge-path.

Claim 2. If two h-products represent the same element in $\mathcal{M}$ and induce edge-paths which are equal modulo backtracking then the h-products are equivalent modulo (P1)-(P10).

Proof. Consider an $h$-product $g=g_{i} h_{i+1} r_{i+1} h_{i+2} r_{i+2}$, where $g_{i}$ is an $h$-product inducing a shorter edge-path $\mathbf{p}$ and the edge-path induced by $g$ has a backtracking at the end. Then $g_{i}\left(v_{0}\right)=v_{i}, g_{i} h_{i+1} r_{i+1}\left(v_{0}\right)=$ 
$v_{i+1}$, and $g_{i} h_{i+1} r_{i+1} h_{i+2} r_{i+2}\left(v_{0}\right)=v_{i}$. Since $r_{i+1}$ and $r_{i+2}$ correspond to the same edge $\left(v_{i}, v_{i+1}\right)$ of a fixed edge type, they must be equal. Clearly the $h$-product $g_{i} h_{i+1} r_{i+1} r_{i+1}$ induces the same edge-path. In particular $g_{i} h_{i+1} r_{i+1}^{2}\left(v_{0}\right)=v_{i}$, hence there exists $h^{\prime} \in H$ such that $g_{i} h_{i+1} r_{i+1}^{2} h^{\prime}=$ $g_{i} h_{i+1} r_{i+1} h_{i+2} r_{i+2}$. Now by Claim 1 the $h$-products are equivalent modulo (P10). But $g_{i} h_{i+1} r_{i+1}^{2} h^{\prime}$ is equivalent modulo (P9) to a shorter $h$-product which induces the edge-path $\mathbf{p}$. Claim 2 follows by induction on the number of backtrackings.

The final set of relations corresponds to the faces of $X$, the closed triangular edge-paths. To every closed edge-path corresponds an $h$-product which is equal in $\mathcal{M}$ to an element of $H$. This gives a relation in $\mathcal{M}$. We shall see that it suffices to consider the triangles modulo the action of $\mathcal{M}$.

Up to an action of $\mathcal{M}$ we may assume that the path starts with $v_{0}$ and that the second vertex is $v_{i, j}$. The third vertex has only one curve which does not belong to $\left\langle\alpha_{1}, \ldots, \alpha_{g}\right\rangle$, because the next vertex is again $v_{0}$.

CAsE 1. Suppose first that $\gamma_{i, j}$ remains fixed in the next simple move. Then some $\alpha_{k}$ is replaced by $\alpha_{j}$. $\partial_{k}$ must be a single hole inside $\gamma_{i, j}$ with $\partial_{-k}$ outside $\gamma_{i, j}$. Applying the product $t_{j-2} t_{j-3} \ldots t_{k}$ we can map $\partial_{k}$ onto $\partial_{j-1}$ leaving $\alpha_{j}$ and $\gamma_{i, j}$ fixed. Thus we may assume that our path, read backwards, has the form

$$
\mathbf{p}_{1}=\left\langle\alpha_{j-1}, \alpha_{j}\right\rangle \rightarrow\left\langle\gamma_{i, j}, \alpha_{j}\right\rangle \rightarrow\left\langle\gamma_{i, j}, \alpha_{j-1}\right\rangle \rightarrow\left\langle\alpha_{j}, \alpha_{j-1}\right\rangle .
$$

This path can be represented by the $h$-product $\left(t_{j-1} r_{i, j}\right)^{3}$. Indeed, for $g=t_{j-1} r_{i, j}$ we have $g\left(\alpha_{j}\right)=\gamma_{i, j}, g\left(\gamma_{i, j}\right)=\alpha_{j-1}, g\left(\alpha_{j-1}\right)=\alpha_{j}$ and $g$ leaves other curves $\alpha_{k}$ fixed.

Since $g^{3}$ represents a closed edge-path it represents an element of $H$ in $\mathcal{M}$. The relation $r_{i, j} * t_{j-1}=t_{j-1}^{-1} * r_{i, j}$ of (P11) is satisfied in $\mathcal{M}$ by Lemma 16(iv). From (P11) we get $g^{3}=t_{j-1} r_{i, j}^{2} t_{j-1} r_{i, j}^{2}$. The last expression is equivalent to an element $f_{1}$ of $H$ modulo (P9). We let $V_{1}=\left(t_{j-1} r_{i, j}\right)^{3} f_{1}^{-1}$. Then $V_{1}$ represents the edge-path $\mathbf{p}_{1}$ and is equal to the identity modulo (P1) $-(\mathrm{P} 11)$.

CASE 2. Suppose now that $\gamma_{i, j}$ changes in the second simple move of a triangular path. Then $g-1$ curves remain fixed at all three vertices of the triangular path and only one curve changes. We call such a path a triangular path of the second kind.

LEMMA 26. Every triangle of the second kind is a sum of triangles of the second kind with at least one edge of type $(1,2)$.

Proof. By the length of an edge $(u, v)$ we mean the minimal number of holes (boundary components) in a component of $S-(u \cup v)$, where $(u \cup v)$ denotes the union of the $g+1$ curves appearing in the cut-systems $u$ and $v$ 
together. The length is an invariant of the edge type. An edge of type $(1,2)$ has length 3 and an edge of any other type is longer.

Consider a triangle of the second kind with all edges of length greater than 3 . We start with a cut-system $\left\langle\alpha_{1}, \ldots, \alpha_{g}\right\rangle$. We have a path of simple moves $\left\langle\alpha_{i}\right\rangle \rightarrow\langle\beta\rangle \rightarrow\langle\gamma\rangle \rightarrow\left\langle\alpha_{i}\right\rangle$. We assume that the edge $\left\langle\alpha_{i}\right\rangle \rightarrow\langle\beta\rangle$ is the shortest edge of the path. All curves are disjoint. The curve $\gamma$ splits $S_{0}$ into two components and $\beta$ lies in one of them. One boundary component corresponding to $\alpha_{i}$, say $\partial_{i}$, lies inside $\beta$ and the other lies outside $\gamma$, in the other component of $S_{0}-\gamma$. We want to split the triangle into smaller, simpler triangles. Every curve disjoint from $\alpha_{1}, \ldots, \alpha_{g}$ is a meridian curve. We choose $\beta_{1}$ inside $\beta$ such that $\beta_{1}$ contains $\partial_{i}$ and exactly one more boundary component $\partial_{j}$ inside $\beta$. Our triangle splits into three triangles $\left\langle\alpha_{i}\right\rangle \rightarrow\langle\beta\rangle \rightarrow$ $\left\langle\beta_{1}\right\rangle \rightarrow\left\langle\alpha_{i}\right\rangle,\left\langle\alpha_{i}\right\rangle \rightarrow\left\langle\beta_{1}\right\rangle \rightarrow\langle\gamma\rangle \rightarrow\left\langle\alpha_{i}\right\rangle$, and $\left\langle\beta_{1}\right\rangle \rightarrow\langle\beta\rangle \rightarrow\langle\gamma\rangle \rightarrow\left\langle\beta_{1}\right\rangle$. The first two have an edge of length 3 and the last one has an edge $\left\langle\beta_{1}\right\rangle \rightarrow\langle\beta\rangle$ shorter than $\left\langle\alpha_{i}\right\rangle \rightarrow\langle\beta\rangle$. The required result follows by induction.

We further investigate the triangles of the second kind with one edge of type $(1,2)$. Up to the action of $\mathcal{M}$ we may assume that the first edge of the path is an edge $\mathbf{e}_{i, j}$, the second is of type $(1,2)$, and the third is the longest. The path has the form $\left\langle\alpha_{j}\right\rangle \rightarrow\left\langle\gamma_{i, j}\right\rangle \rightarrow\langle\gamma\rangle \rightarrow\left\langle\alpha_{j}\right\rangle$.

The curve $\gamma$ is disjoint from $\gamma_{i, j}$, it is separated from $\gamma_{i, j}$ by a single hole $\partial_{k}, k \neq j$, and the edge represented by the simple move $\langle\gamma\rangle \rightarrow\left\langle\alpha_{j}\right\rangle$ is the longest edge of the path. It follows that $\gamma$ must lie outside $\gamma_{i, j}$ and up to the action of $H_{i, j}$ we may assume that $\gamma=\gamma_{i, j+1}$, or $\gamma=\gamma_{i-1, j}\left(\gamma_{-1, j}\right.$ if $i=1)$. For each of these triangles we have a relation in $\mathcal{M}$. Some of these relations are listed in (P12) and the other relations follow from these.

Consider first the case $i=1$ and the path

$$
\mathbf{p}_{2}=\left\langle\alpha_{j}\right\rangle \rightarrow\left\langle\gamma_{1, j+1}\right\rangle \rightarrow\left\langle\gamma_{1, j}\right\rangle \rightarrow\left\langle\alpha_{j}\right\rangle .
$$

Let

$$
h_{2}=k_{j}^{-1} t_{j-1}^{-1} t_{j-2}^{-1} \ldots t_{1}^{-1} k_{j} k_{j-1} \ldots k_{2} .
$$

We want to prove that

$$
g=k_{j}^{-1} r_{1, j+1} k_{j} h_{2} r_{1,2} h_{2}^{-1} s_{j}^{-1} r_{1, j}
$$

represents the edge-path $\mathbf{p}_{2}$. Recall that $r_{1, j}\left(\alpha_{j}\right)=\gamma_{1, j}, k_{j}\left(\alpha_{j}\right)=t_{j}\left(\alpha_{j}\right)=$ $\alpha_{j+1}$ and $k_{j}\left(\beta_{j}\right)=\beta_{j+1}$ for all $j$. It follows that $h_{2}\left(\alpha_{2}\right)=\alpha_{j}, h_{2}\left(\beta_{2}\right)=\beta_{j}$. Also $k_{j}^{-1} * r_{1, j+1}=b_{j} a_{j} c_{1, j+1} b_{j}$ by Lemma 15 . It is also easy to check that $h_{2}\left(\delta_{1,2}\right)=\delta_{j, j+1}$. Therefore $h_{2} * r_{1,2}=b_{j} a_{j} d_{j, j+1} b_{j}$. We get $g=$ $b_{j} a_{j} c_{1, j+1} b_{j} b_{j} d_{j, j+1} c_{1, j} b_{j}$. We have to check that

$$
\begin{gathered}
k_{j}^{-1} r_{1, j+1}\left(v_{0}\right)=\left\langle\alpha_{1}, \ldots, \alpha_{j-1}, \gamma_{1, j+1}, \alpha_{j+1}, \ldots, \alpha_{g}\right\rangle, \\
k_{j}^{-1} r_{1, j+1} k_{j} b_{j} a_{j} d_{j, j+1} b_{j}\left(v_{0}\right)=\left\langle\alpha_{1}, \ldots, \alpha_{j-1}, \gamma_{1, j}, \alpha_{j+1}, \ldots, \alpha_{g}\right\rangle, g\left(v_{0}\right)=v_{0} .
\end{gathered}
$$


Everything follows from what we already know with the exception of the following two facts, which have to be checked by drawings:

$$
k_{j}^{-1} r_{1, j+1} k_{j} b_{j} a_{j} d_{j, j+1} b_{j}\left(\alpha_{j}\right)=\gamma_{1, j} \quad \text { and } \quad g\left(\alpha_{j}\right)=\alpha_{j} .
$$

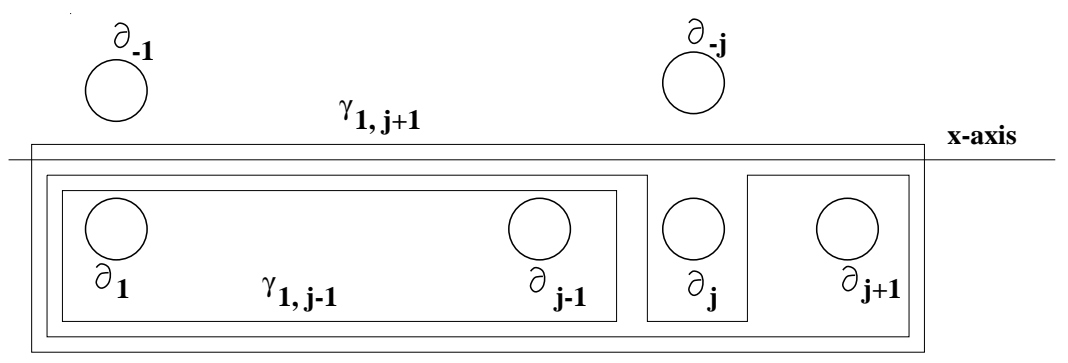

Fig. 11. The first lantern relation for triangles of type 2

We want to find an element of $H$ which is equal to $g$ in $\mathcal{M}$. Consider the domain in $S_{0}$ bounded by the four curves $\gamma_{1, j+1}, \gamma_{1, j-1}, \partial_{j}$ and $\partial_{j+1}$ (see Figure 11). By the lantern relation (Lemma 17)

$$
c_{1, j+1} c_{1, j-1} a_{j} a_{j+1}=d_{j, j+1} c_{1, j}\left(t_{j} c_{1, j} t_{j}^{-1}\right)
$$

$\left(t_{j} c_{1, j} t_{j}^{-1}=d_{I}\right.$ with $\left.I=\{1,2, \ldots, j-1, j+1\}\right)$. Therefore $d_{j, j+1} c_{1, j}=$ $a_{j} c_{1, j+1} c_{1, j-1} a_{j+1} t_{j} c_{1, j}^{-1} t_{j}^{-1}$. Also $c_{1, j-1}, a_{j+1}$ and $t_{j} c_{1, j}^{-1} t_{j}^{-1}$ commute with $b_{j}$, by Lemma 16 . Hence

$$
\begin{aligned}
g & =b_{j} a_{j} c_{1, j+1} b_{j} b_{j} d_{j, j+1} c_{1, j} b_{j} \\
& =\left(b_{j} a_{j} c_{1, j+1} b_{j}\right)^{2} a_{j+1} c_{1, j-1} t_{j} c_{1, j}^{-1} t_{j}^{-1}=k_{j}^{-1} r_{1, j+1}^{2} k_{j} a_{j+1} c_{1, j-1} t_{j} c_{1, j}^{-1} t_{j}^{-1} \\
& =k_{j}^{-1} s_{j+1} c_{1, j+1} s_{j+1} c_{1, j+1}^{-1} k_{j} a_{j+1} c_{1, j-1} t_{j} c_{1, j}^{-1} t_{j}^{-1}=f_{2} \in H .
\end{aligned}
$$

From this equation we can compute $r_{1, j+1}$ in terms of $r_{1, k}, k<j+1$, and the generators of $H$. We get a formula from (P12). The relation $g=f_{2}$ becomes trivial modulo (P1)-(P12). If we let $V_{2}=g f_{2}^{-1}$ then $V_{2}$ is an $h$-product which represents the path $\mathbf{p}_{2}$ and is equal to the identity modulo (P1) $-(\mathrm{P} 12)$.

Consider now the edge-path

$$
\left.\mathbf{p}_{3}=\left\langle\alpha_{j}\right\rangle \rightarrow\left\langle\gamma_{i, j}\right\rangle \rightarrow \gamma_{i-1, j}\right\rangle \rightarrow\left\langle\alpha_{j}\right\rangle
$$

(or $\mathbf{p}_{3}=\left\langle\alpha_{j}\right\rangle \rightarrow\left\langle\gamma_{1, j}\right\rangle \rightarrow\left\langle\gamma_{-1, j}\right\rangle \rightarrow\left\langle\alpha_{j}\right\rangle$ if $i=1$ ). We let

$$
h_{3}=s_{1-i} t_{-i}^{-1} t_{-1-i}^{-1} \ldots t_{2}^{-1} t_{1}^{-1} k_{j-1} k_{j-2} \ldots k_{2}
$$

$\left(h_{3}=s_{1} k_{j-1} k_{j-2} \ldots k_{2}\right.$ if $\left.i=1\right)$.

We consider in detail the case $i<0$. The case $i=1$ requires only a suitable change of indices. We want to prove that

$$
g=r_{i, j} s_{j}^{-1} h_{3} r_{1,2} h_{3}^{-1} r_{i-1, j}
$$


is an $h$-product representing $\mathbf{p}_{3}$. It is easy to check that $h_{3}\left(\beta_{2}\right)=\beta_{j}$, $h_{3}\left(\alpha_{2}\right)=\alpha_{j}$ and $h_{3}\left(\delta_{1,2}\right)=\gamma$, where $\gamma$ is the curve in Figure 12. By Lemma $15, h_{3} r_{1,2} h_{3}^{-1}=b_{j} a_{j} c b_{j}$, where $c$ is the twist around $\gamma$. Thus $g$ is equal in $\mathcal{M}$ to $g=b_{j} c_{i, j} c b_{j} b_{j} a_{j} c_{i-1, j} b_{j}$. We have to check that

$$
\begin{aligned}
r_{i, j}\left(v_{0}\right) & =\left\langle\alpha_{1}, \ldots, \alpha_{j-1}, \gamma_{i, j}, \alpha_{j+1}, \ldots, \alpha_{g}\right\rangle, \\
b_{j} c_{i, j} c b_{j}\left(v_{0}\right) & =\left\langle\alpha_{1}, \ldots, \alpha_{j-1}, \gamma_{i-1, j}, \alpha_{j+1}, \ldots, \alpha_{g}\right\rangle,
\end{aligned}
$$

and $g\left(v_{0}\right)=v_{0}$. Everything follows from relations which we already know with the exception of the following two facts, which have to be checked by drawings:

$$
b_{j} c_{i, j} c b_{j}\left(\alpha_{j}\right)=\gamma_{i-1, j} \quad \text { and } \quad g\left(\alpha_{j}\right)=\alpha_{j} .
$$

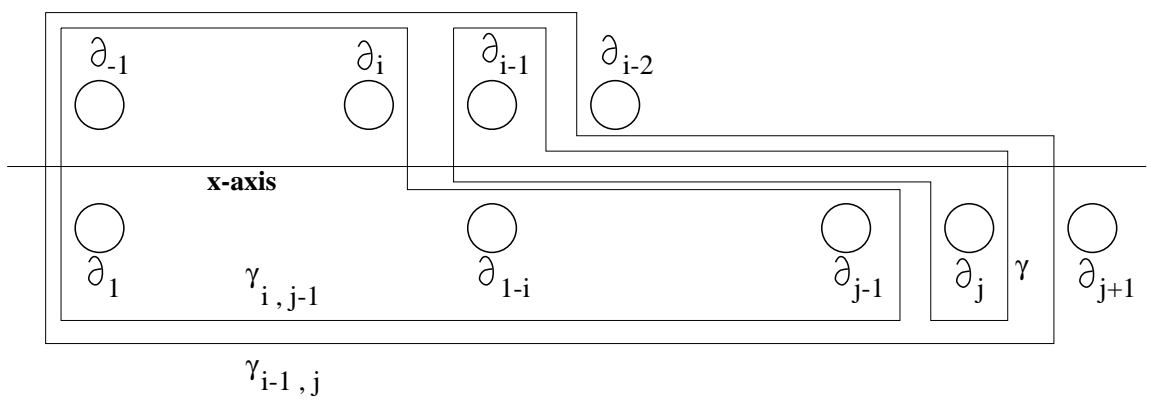

Fig. 12. The second lantern relation for triangles of type 2

We now want to find an element of $H$ which is equal to $g$ in $\mathcal{M}$. Consider the domain in $S_{0}$ bounded by the four curves $\gamma_{i-1, j}, \gamma_{i, j-1}, \partial_{i-1}$ and $\partial_{j}$ (see Figure 12). By the lantern relation (Lemma 17)

$$
c_{i-1, j} c_{i, j-1} a_{i-1} a_{j}=c_{i-1, j-1} c_{i, j} c .
$$

Therefore $c_{i, j} c=c_{i-1, j-1}^{-1} c_{i, j-1} a_{i-1} c_{i-1, j} a_{j}$. Moreover $c_{i-1, j-1}, c_{i, j-1}$ and $a_{i-1}$ commute with $b_{j}$ by Lemma 16 . Therefore

$$
\begin{aligned}
g & =b_{j} c_{i, j} c b_{j} b_{j} a_{j} c_{i-1, j} b_{j}=c_{i-1, j-1}^{-1} c_{i, j-1} a_{i-1} r_{i-1, j}^{2} \\
& =c_{i-1, j-1}^{-1} c_{i, j-1} a_{i-1} s_{j} c_{i-1, j} s_{j} c_{i-1, j}^{-1}=f_{3} \in H .
\end{aligned}
$$

From this equation we can compute $r_{i-1, j}$ in terms of $r_{k, j}, j-k<j+1-i$, and the generators of $H$. We get a relation from (P12). The relation $g=f_{3}$ becomes trivial modulo (P1)-(P12). If we let $V_{3}=g f_{3}^{-1}$ then $V_{3}$ is an $h$-product which represents the path $\mathbf{p}_{3}$ and is equal to the identity modulo (P1)-(P12).

Consider now the diagram in Figure 13. Every triangle in this diagram has at least one edge of type $(1,2)$. (In the case of $i=-1$ we should replace $i+1$ by 1.) By the previous discussion every triangle with an edge of type $(1,2)$ is equivalent modulo the action of $\mathcal{M}$ to the outside triangle 


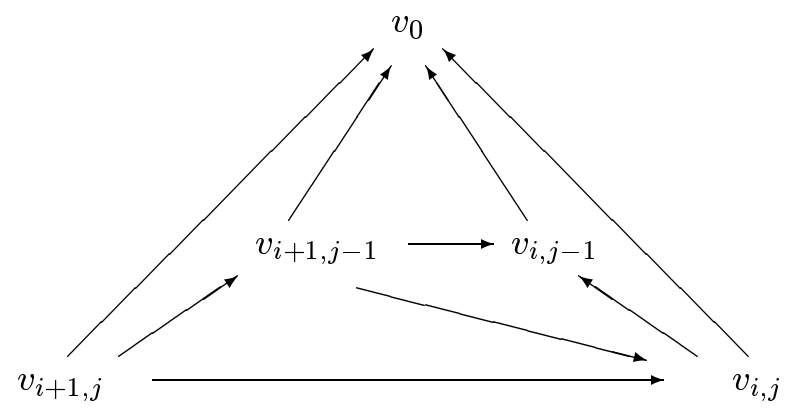

Fig. 13. Reduction for triangular paths

on the diagram or to the triangle on the right hand side of the diagram. The outside triangle and the right hand side triangle have the longest edge of length $j-i+2(j+2$ if $i=-1)$ and the other triangles have the longest edge of a smaller length.

If $i \neq 1$ then the outer triangle is represented by an $h$-product $V_{3}$ which is trivial modulo (P1)-(P12), by the definition of $r_{i, j}$. The triangle on the right hand side is a sum of the remaining triangles. If $i=1$ then the triangle on the right hand side is represented by an $h$-product $V_{2}$ which is trivial modulo (P1)-(P12), by the definition of $r_{i, j}$. The outside triangle is a sum of the remaining triangles.

We can now complete the proof of Theorem 18 . Let $W$ be a word in the generators of $\mathcal{M}$ which represents the identity in $\mathcal{M}$. It has the form of an $h$-product $g$ which represents a closed edge-path $\mathbf{p}$. We want to prove that $g$ is equivalent to the identity modulo (P1)-(P12). By Proposition 1 and the above discussion, $\mathbf{p}$ is equal modulo backtracking to a sum of paths of the form $\mathbf{q}_{1} \mathbf{q}_{2} \mathbf{q}_{1}^{-1}$, where $\mathbf{q}_{1}$ starts at $v_{0}$ and $\mathbf{q}_{2}$ is equivalent modulo the action of $\mathcal{M}$ to one of the special paths $\mathbf{p}_{1}, \mathbf{p}_{2}, \mathbf{p}_{3}$ or their inverses. By Claims 1 and 2 it suffices to assume that $g$ itself represents the edge-path $\mathbf{q}_{1} \mathbf{q}_{2} \mathbf{q}_{1}^{-1}$.

Let $g_{1}$ be an $h$-product representing $\mathbf{q}_{1}$ and let $f$ be an element of $\mathcal{M}$ such that $\mathbf{q}_{2}=f\left(\mathbf{p}_{i}\right)$. Then $g_{1}\left(v_{0}\right)=f\left(v_{0}\right)$ is the first vertex of $\mathbf{q}_{2}$, hence $g_{1}^{-1} f=h \in H$. If $V_{i}$ is the $h$-product representing $\mathbf{p}_{i}$ then the $h$-product $g_{1} h V_{i}$ represents $\mathbf{q}_{1} \mathbf{q}_{2}$ and there exists an $h$-product $g_{1} h V_{i} g_{3}$ which represents the identity in $\mathcal{M}$ and represents the edge-path $\mathbf{q}_{1} \mathbf{q}_{2} \mathbf{q}_{1}^{-1}$. Clearly $g_{1} h g_{3}$ represents the edge-path $\mathbf{q}_{1} \mathbf{q}_{1}^{-1}$ null-homotopic by backtracking. By Claim $1, g$ is equivalent to $g_{1} h V_{i} g_{3}$, which is equivalent to $g_{1} h g_{3}$, which is equivalent to the identity modulo (P1)-(P12).

A path inverse to $\mathbf{p}_{i}$ is represented by some other $h$-product $V_{i}^{\prime}$ but then $V_{i} V_{i}^{\prime}$ represents a path contractible by backtracking. Thus $V_{i} V_{i}^{\prime}$ is equivalent to the identity modulo $(\mathrm{P} 1)-(\mathrm{P} 10)$ and $V_{i}$ is equivalent to the identity so $V_{i}^{\prime}$ is also equivalent to the identity. This concludes the proof of Theorem 18. 


\section{References}

[1] P. Bergau und J. Mennicke, Über topologische Abbildungen der Bretzelfläche vom Geschlecht 2, Math. Z. 74 (1960), 414-435.

[2] J. S. Birman, Braids, Links, and Mapping Class Groups, Ann. of Math. Stud. 82, Princeton Univ. Press, 1974.

[3] J. S. Birman and H. Hilden, On mapping class groups of closed surfaces as covering spaces, in: Advances in the Theory of Riemann Surfaces, Ann. of Math. Stud. 66, Princeton Univ. Press, 1971, 81-115.

[4] M. Dehn, Die Gruppe der Abbildungsklassen, Acta Math. 69 (1938), 135-206.

[5] D. B. A. Epstein, Curves on 2-manifolds and isotopies, ibid. 115 (1966), 83-107.

[6] J. Harer, The second homology group of the mapping class group of an orientable surface, Invent. Math. 72 (1983), 221-239.

[7] A. Hatcher and W. Thurston, A presentation for the mapping class group of a closed orientable surface, Topology 19 (1980), 221-237.

[8] M. Heusner, Eine Präsentation der Abbildungsklassengruppe einer geschlossenen, orientierbaren Fläche, Diplomarbeit, University of Frankfurt.

[9] S. Humphries, Generators for the mapping class group, in: Topology of LowDimensional Manifolds, Lecture Notes in Math. 722, Springer, 1979, 44-47.

[10] D. Johnson, Homeomorphisms of a surface which act trivially on homology, Proc. Amer. Math. Soc. 75 (1979), 119-125.

[11] F. Laudenbach, Présentation du groupe de difféotopies d'une surface compacte orientable, in: Travaux de Thurston sur les surfaces, Astérisque 66-67 (1979), 267282.

[12] W. B. R. Lickorish, A finite set of generators for the homeotopy group of a 2manifold, Proc. Cambridge Philos. Soc. 60 (1964), 769-778.

[13] S. Suzuki, On homeomorphisms of a 3-dimensional handlebody, Canad. J. Math. 29 (1977), 111-124.

[14] B. Wajnryb, A simple presentation for the mapping class group of an orientable surface, Israel J. Math. 45 (1983), 157-174.

Department of Mathematics

Technion

32000 Haifa, Israel

E-mail: wajnryb@techunix.technion.ac.il

Received 2 September 1997;

in revised form 5 January 1998 and 22 June 1998 